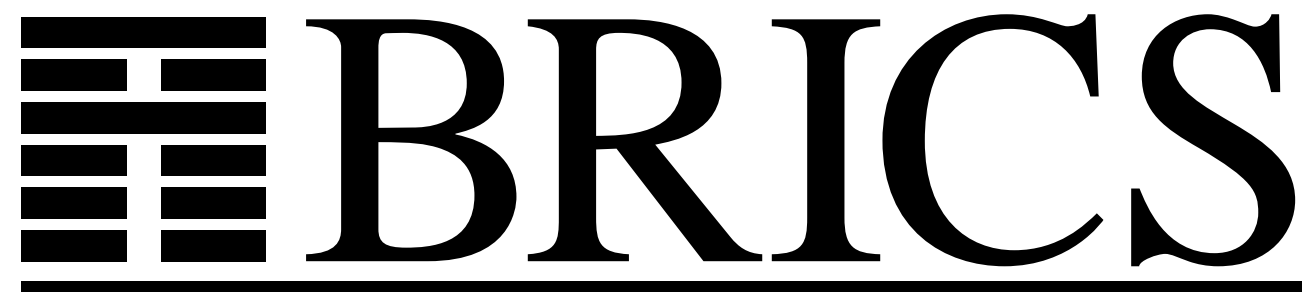

Basic Research in Computer Science

\title{
Compact Representations of BL-Algebras
}

\author{
Antonio Di Nola \\ Laurenţiu Leuştean
}


Copyright (c) 2002, $\quad$ Antonio Di Nola \& Laurenţiu Leuştean.

BRICS, Department of Computer Science

University of Aarhus. All rights reserved.

Reproduction of all or part of this work is permitted for educational or research use on condition that this copyright notice is included in any copy.

See back inner page for a list of recent BRICS Report Series publications. Copies may be obtained by contacting:

\author{
BRICS \\ Department of Computer Science \\ University of Aarhus \\ Ny Munkegade, building 540 \\ DK-8000 Aarhus C \\ Denmark \\ Telephone: +4589423360 \\ Telefax: $\quad+4589423255$ \\ Internet: BRICS@brics.dk
}

BRICS publications are in general accessible through the World Wide Web and anonymous FTP through these URLs:

http://www.brics.dk

ftp: / / ftp.brics.dk

This document in subdirectory RS / 02/23/ 


\title{
Compact representations of BL-algebras
}

\author{
Antonio Di Nola* and Laurenţiu Leuştean** \\ * Università di Salerno, Facoltà di Scienze, Dipartimento di Matematica e Informatica, \\ Via S. Allende, 84081 Baronissi, Salerno, Italy \\ E-mail: dinola@ds.unina.it \\ ** National Institute for Research and Developement in Informatics, \\ 8-10 Averescu Avenue, 71316, Bucharest, 1, Romania, \\ E-mail: leo@u3.ici.ro
}

\begin{abstract}
In this paper we define sheaf spaces of BL-algebras (or BL-sheaf spaces), we study completely regular and compact BL-sheaf spaces and compact representations of BL-algebras and, finally, we prove that the category of non-trivial BL-algebras is equivalent with the category of compact local BL-sheaf spaces.
\end{abstract}

\section{Introduction}

BL-algebras are the algebraic structures for Hájek's Basic Logic [11]. The main example of a BL-algebra is the interval $[0,1]$ endowed with the structure induced by a $t$-norm.

In this paper we study compact representations of BL-algebras, following techniques used for ringed spaces by Mulvey [14, 13, 15]. In [13], Mulvey extended the concepts of complete regularity and compactness from topological spaces to ringed spaces and proved a compactness theorem for completely regular ringed spaces generalizing the Gelfand-Kolmogoroff criterion concerning maximal ideals in the ring $\mathbf{R}(X)$ of continuous real functions on a completely regular space $X$ [8]. In [14], Mulvey introduced compact representations of rings, showing that they are exactly those representations of rings that establish an equivalence of categories of modules. Using compact representations, Mulvey extended the Gelfand duality between the categories of compact spaces and commutative $C^{*}$ algebras to Gelfand rings [15].

Gelfand rings are characterized by a property that can be formulated in terms of universal algebra, namely that each prime ideal is contained in a unique maximal ideal. Universal algebras with this property and their Gelfand representations 
were studied by Georgescu and Voiculescu [10] and, in a lattice-theoretical setting, by Simmons [18].

MV-algebras [1] and BL-algebras are classes of algebras that also satisfy this property. Hence, the problem of obtaining similar results for these structures is natural. Filipoiu and Georgescu [7] proved that the category of MV-algebras is equivalent with the category of compact sheaf spaces of MV-algebras with local stalks.

In the present paper, we give an answer for this problem in the case of BLalgebras. In different classes of problems, sheaf representations of universal algebras are very useful since they reduce the study of algebras to the study of the stalks, which usually have a better known structure. In the case of our compact representations, the stalks are local BL-algebras, introduced and studied by Turunen and Sessa [22].

In the first section of the paper we recall some facts about BL-algebras and we study some special filters of BL-algebras, used in the sequel. In Section 2, we define sheaf spaces of BL-algebras (or BL-sheaf spaces), BL-algebras of global sections, morphisms of BL-sheaf spaces and other notions related with sheaf theory.

In the next section we define and study completely regular and compact BLsheaf spaces and we prove the compactness theorem.

In the following section we remind some general results concerning sheaf representations of BL-algebras and we study a special kind of representations, namely compact representations. We prove that any compact representation of a BLalgebra arises canonically from a family of filters of the BL-algebra satisfying certain conditions.

Finally, in the last section of the paper we prove that the functor from the category of compact local BL-sheaf spaces to the category of non-trivial BLalgebras, obtained by assigning to each BL-sheaf space the BL-algebra of global sections determines an equivalence between these categories.

\section{BL-algebras}

A $B L$-algebra [11] is an algebra $(A, \wedge, \vee, \odot, \rightarrow, 0,1)$ with four binary operations $\wedge, \vee, \odot, \rightarrow$ and two constants 0,1 such that:

(i) $(A, \wedge, \vee, 0,1)$ is a bounded lattice;

(ii) $(A, \odot, 1)$ is a commutative monoid;

(iii) $\odot$ and $\rightarrow$ form an adjoint pair, i.e. $c \leq a \rightarrow b$ iff $a \odot c \leq b$ for all $a, b, c \in A$;

(iv) $a \wedge b=a \odot(a \rightarrow b)$;

(v) $(a \rightarrow b) \vee(b \rightarrow a)=1$.

A BL-algebra $A$ is nontrivial iff $0 \neq 1$.

For any BL-algebra $A$, the reduct $L(A)=(A, \wedge, \vee, 0,1)$ is a bounded distributive lattice. 
A BL-chain is a linear BL-algebra, i.e. a BL-algebra such that its lattice order is total.

For any $a \in A$, we define $a^{-}=a \rightarrow 0$. We denote the set of natural numbers by $\omega$. We define $a^{0}=1$ and $a^{n}=a^{n-1} \odot a$ for $n \in \omega-\{0\}$. The order of $a \in A$, in symbols $\operatorname{ord}(a)$, is the smallest $n \in \omega$ such that $a^{n}=0$. If no such $n$ exists, then $\operatorname{ord}(a)=\infty$.

The following properties hold in any BL-algebra $A$ and will be used in the sequel:

(1.1) $a \odot b \leq a \wedge b \leq a, b$

(1.2) $a \leq b$ implies $a \odot c \leq b \odot c$

(1.3) $0 \rightarrow a=1$ and $1 \rightarrow a=a$

(1.4) $a \rightarrow b=1$ iff $a \leq b$

(1.5) $a \odot b=0$ iff $a \leq b^{-}$

(1.6) $a \odot a^{-}=0$

(1.7) $a \rightarrow(b \rightarrow c)=(a \odot b) \rightarrow c$

(1.8) $(a \wedge b)^{-}=a^{-} \vee b^{-}$and $(a \vee b)^{-}=a^{-} \wedge b^{-}$

(1.9) $1^{-}=0$ and $0^{-}=1$

(1.10) $a^{-}=1$ iff $a=0$

(1.11) $a \vee b=1$ implies $a^{n} \vee b^{n}=1$ for any $n \in \omega$

Let $A$ be a BL-algebra. A filter of $A$ is a nonempty set $F \subseteq A$ such that for all $a, b \in A$,

(i) $a, b \in F$ implies $a \odot b \in F$;

(ii) $a \in F$ and $a \leq b$ imply $b \in F$.

A filter $F$ of $A$ is proper iff $F \neq A$.

By (1.1) it is obvious that any filter of $A$ is also a filter of the lattice $L(A)$. A proper filter $P$ of $A$ is called prime provided that it is prime as a filter of $L(A)$ : $a \vee b \in P$ implies $a \in P$ or $b \in P$.

A proper filter $M$ of $A$ is called maximal (or ultrafilter) if it is not contained in any other proper filter.

We shall denote by $\operatorname{Spec}(A)$ the set of prime filters of $A$ and by $\operatorname{Max}(A)$ the set of maximal filters of $A$. Let us remind some properties of filters that will be used in the sequel.

Proposition 1.1 ([21, Proposition 8]

If $A$ is a nontrivial BL-algebra, then any proper filter of $A$ can be extended to a maximal filter.

Proposition 1.2 [21, Proposition 6]

Let $P$ be a prime filter of a nontrivial BL-algebra $A$. Then the set $\mathcal{F}=\{F \mid P \subseteq F$ and $F$ is a proper filter of $A\}$

is linearly ordered with respect to set-theoretical inclusion.

Proposition 1.3 [6, Proposition 1.6]

If $A$ is a nontrivial BL-algebra, then any prime filter of $A$ is contained in a unique maximal filter. 
Proposition 1.4 [21, Proposition 7]

Any maximal filter of $A$ is a prime filter of $A$.

Let $X \subseteq A$. The filter of $A$ generated by $X$ will be denoted by $\langle X\rangle$. We have that $\langle\bar{\emptyset}\rangle=\{1\}$ and $\left\langle X>=\left\{a \in A \mid x_{1} \odot \cdots \odot x_{n} \leq a\right.\right.$ for some $n \in \omega-\{0\}$ and some $\left.x_{1}, \cdots, x_{n} \in X\right\}$ if $\emptyset \neq X \subseteq A$. For any $a \in A,\langle a\rangle$ denotes the principal filter of $A$ generated by $\{a\}$. Then, $<a\rangle=\left\{b \in A \mid a^{n} \leq b\right.$ for some $n \in \omega-\{0\}\}$.

Lemma 1.5 Let $F, G$ be filters of $A$. Then $<F \cup G>=\{a \in A \mid b \odot c \leq a$ for some $b \in F, c \in G\}$

Proposition 1.6 Let $\mathcal{F}(A)$ be the set of filters of $A$. Then $(\mathcal{F}(A), \subseteq)$ is a complete lattice. For every family $\left\{F_{i}\right\}_{i \in I}$ of filters of $A$, we have that $\wedge_{i \in I} F_{i}=\cap_{i \in I} F_{i}$ and $\vee_{i \in I} F_{i}=<\cup_{i \in I} F_{i}>$.

With any filter $F$ of $A$ we can associate a congruence relation $\sim_{F}$ on $A$ by defining

$$
a \sim_{F} b \text { iff } a \rightarrow b \in F \text { and } b \rightarrow a \in F \text { iff }(a \rightarrow b) \odot(b \rightarrow a) \in F .
$$

For any $a \in A$, let $a / F$ be the equivalence class $a / \sim_{F}$. If we denote by $A / F$ the quotient set $A / \sim_{F}$, then $A / F$ becomes a BL-algebra with the natural operations induced from those of $A$.

\section{Proposition $1.7[11]$}

Let $F$ be a filter of $A$ and $a, b \in A$.

(i) $a / F=1 / F$ iff $a \in F$;

(ii) $a / F=0 / F$ iff $a^{-} \in F$;

(iii) for all $a, b \in A$, $a / F \leq b / F$ iff $a \rightarrow b \in F$;

(iv) $A / F$ is a BL-chain iff $F$ is prime.

If $h: A \rightarrow B$ is a BL-morphism, then the kernel of $h$ is the set $\operatorname{Ker}(h)=\{a \in$ $A \mid h(a)=1\}$. It is easy to see that

Proposition 1.8 Let $h: A \rightarrow B$ be a BL-morphism. If $G$ is a (proper, prime) filter of $B$, then $h^{-1}(G)$ is a (proper, prime) filter of $A$. Thus, in particular, $\operatorname{Ker}(h)$ is a proper filter of $A$.

Lemma 1.9 [5, Proposition 1.13]

Let $A$ be a nontrivial BL-algebra and $M$ a proper filter of $A$. The following are equivalent:

(i) $M$ is maximal;

(ii) for any $x \in A$, $x \notin M$ implies $\left(x^{n}\right)^{-} \in M$ for some $n \in \omega$. 
Proposition 1.10 Let $h: A \rightarrow B$ be a BL-morphism. If $N$ is a maximal filter of $B$, then $h^{-1}(N)$ is a maximal filter of $A$.

Proof: By Proposition 1.8, we have that $h^{-1}(N)$ is a proper filter of $A$. In order to get that it is maximal, we shall apply Lemma 1.9. Let $x \in A$ such that $x \notin h^{-1}(N)$, hence $h(x) \notin N$. Since $N$ is a maximal filter of $B$, there is $n \in \omega$ such that $\left(h(x)^{n}\right)^{-} \in N$, that is $h\left(\left(x^{n}\right)^{-}\right) \in N$, since $h$ is a homomorphism of BL-algebras. We have got that $\left(x^{n}\right)^{-} \in h^{-1}(N)$.

For any filter $F$ of $A$, let us denote by []$_{F}$ the natural homomorphism from $A$ onto $A / F$, defined by []$_{F}(a)=a / F$ for any $a \in A$. Then $F=\operatorname{Ker}\left([]_{F}\right)$.

Proposition 1.11 [9, Proposition 1.12]

Let $A$ be a BL-algebra and $F$ a filter of $A$.

(i) the map $G \stackrel{\alpha}{\mapsto}[]_{F}(G)$ is an inclusion-preserving bijective correspondence between the filters of $A$ containing $F$ and the filters of $A / F$. The inverse map is also inclusion-preserving;

(ii) $G$ is a proper filter of $A$ containing $F$ iff []$_{F}(G)$ is a proper filter of $A / F$. Hence, there is a bijection between the proper filters of $A$ containing $F$ and the proper filters of $A / F$;

(iii) there is a bijection between the maximal filters of $A$ containing $F$ and the maximal filters of $A / F$.

Following [22], a BL-algebra $A$ is local if it has a unique maximal filter.

Proposition 1.12 [22] Let $A$ be a local BL-algebra. Then its unique maximal filter is

$$
\{a \in A \mid \operatorname{ord}(a)=\infty\} \text {. }
$$

Proposition 1.13 Let $P$ be a proper filter of $A$. The following are equivalent:

(i) $A / P$ is a local BL-algebra;

(ii) $P$ is contained in a unique maximal filter of $A$.

Proof: Apply [9, Proposition 2.6 ], and [9, Proposition 2.8 ].

Let $A$ be a nontrivial BL-algebra. The prime spectrum of $A$ is the set $\operatorname{Spec}(A)$ of prime filters of $A$, endowed with the Zariski topoloy, of which the subsets of the form

$$
D(a)=\{P \in \operatorname{Spec}(A) \mid a \notin P\} \text { for } a \in A
$$

form a basis of open sets.

The maximal spectrum of $A$ is the subspace $\operatorname{Max}(A)$ of $\operatorname{Spec}(A)$ consisting of the maximal filters of $A$ with the induced topology. The subsets

$$
d(a)=D(A) \cap \operatorname{Max}(A)=\{M \in \operatorname{Max}(A) \mid a \notin M\}, a \in A
$$

form a basis for the topology of the maximal spectrum. Then $\operatorname{Spec}(A)$ is a compact topological space and $\operatorname{Max}(A)$ is compact and Hausdorff [12]. 
In the sequel, we shall remind some facts concerning the reticulation of a BLalgebra $A$. For details see [12].

Let us define a binary relation $\equiv$ on $A$ by

$$
a \equiv b \text { iff } D(a)=D(b) .
$$

Then $\equiv$ is an equivalence relation on $A$ compatible with the operations $\odot, \wedge$ and $\vee$. For $a \in A$ let us denote by $[a]$ the class of $a \in A$ with respect to $\equiv$. The bounded distributive lattice $\beta(A)=(A / \equiv, \vee, \wedge,[0],[1])$ is called the reticulation of the BL-algebra $A$.

If $h: A \rightarrow B$ is a homomorphism of BL-algebras, then $\beta(h): \beta(A) \rightarrow \beta(B)$, defined by $\beta(h)(a)=[h(a)]$, is a homomorphism of bounded distributive lattices. It follows that we can define a functor $\beta$ from the category of nontrivial BLalgebras to the category of bounded distributive lattices. The functor $\beta$ is called the reticulation functor.

If $F$ is a filter of $A$, then $\beta(F)=\{[a] \mid a \in A\}$ is a filter of the lattice $\beta(A)$ and the mapping $F \mapsto \beta(F)$ is an isomorphism between the lattice $\mathcal{F}(A)$ of filters of $A$ and the lattice $\mathcal{F}(\beta(A))$ of filters of $\beta(A)$. If $P \in \operatorname{Spec}(A)$, then $\beta(P)$ is a prime filter of $\beta(A)$ and the mapping $P \mapsto \beta(P)$ is a homeomorphism between $\operatorname{Spec}(A)$ and $\operatorname{Spec}(\beta(A))$. Similarly, $\operatorname{Max}(A)$ is homeomorphic to $\operatorname{Max}(\beta(A))$.

Let us remind that a bounded distributive lattice $L$ is called normal ([23], [2]) if each prime ideal of $L$ contains a unique minimal prime ideal.

Proposition 1.14 [12, Proposition 3.14]

For any nontrivial BL-algebra $A, \beta(A)$ is a normal lattice.

To any prime filter $P$ of a bounded distributive lattice or a BL-algebra $A$ we associate the set

$$
O(P)=\{a \in A \mid a \vee b=1 \text { for some } b \notin P\} .
$$

Then it is easy to see that $O(P)$ is a proper filter of $A$ such that $O(P) \subseteq P$.

We have the following characterization of normal lattices

Proposition 1.15 [16, Theorem 3]

Let $L$ be a bounded distributive lattice. The following are equivalent:

(i) $L$ is normal;

(ii) for any maximal filter $M$ of $L, M$ is the unique maximal filter that contains $O(M)$.

Lemma 1.16 For any maximal filter $M$ of $A$,

$$
\beta(O(M))=O(\beta(M)) \text {. }
$$

Proof: In the proof, we use that for all $a \in A,[a]=[1]$ iff $a=1$ and for each maximal filter $M$ of $A, a \in M$ iff $[a] \in \beta(M)[12]$.

"ᄃ" Let $[a] \in \beta(O(M))$, so there is $b \in O(M)$ such that $[a]=[b]$. Since $b \in O(M)$, there is $c \notin M$ such that $b \vee c=1$. It follows that $[a] \vee[c]=[b] \vee[c]=[1]$ and $[c] \notin \beta(M)$. Hence, $[a] \in O(\beta(M)$. 


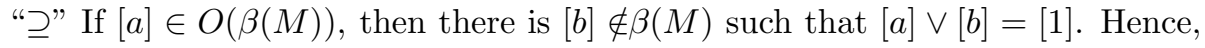
there is $b \notin M$ such that $a \vee b=1$, that is $a \in O(M)$, so $[a] \in \beta(O(M))$.

Proposition 1.17 Let $A$ be a nontrivial BL-algebra. Then

(i) for any maximal filter $M$ of $A, M$ is the unique maximal filter that contains $O(M)$;

(ii) for any distinct maximal filters $M, N$ of $A, O(M) \vee O(N)=A$;

(iii) $A / O(M)$ is local for any $M \in \operatorname{Max}(A)$.

Proof: (i) Apply Proposition 1.15, Lemma 1.16 and the properties of the reticulation of $A$.

(ii) Suppose that $O(M) \vee O(N)$ is a proper filter of $A$. Then, using Proposition 1.1 , we get a contradiction to (i).

(iii) Apply (i) and Proposition 1.13.

Proposition 1.18 [4, Proposition 4.36]

Let $A$ be a nontrivial BL-algebra. Then $\bigcap_{M \in \operatorname{Max}(A)} O(M)=\{1\}$.

\section{BL-sheaf spaces. Definitions and first proper- ties}

A sheaf space of $B L$-algebras (or a $B L$-sheaf space) is a triple $(F, p, X)$ such that the following properties are satisfied:

(i) $F$ and $X$ are topological spaces;

(ii) $p: F \rightarrow X$ is a local homeomorphism from $\mathrm{F}$ onto $\mathrm{X}$;

(iii) for each $x \in X, p^{-1}(\{x\})=F_{x}$ is a nontrivial BL-algebra with operations denoted by $\vee_{x}, \wedge_{x}, \odot_{x}, \rightarrow_{x}, 0_{x}, 1_{x}$;

(iv) the functions $(a, b) \mapsto a \vee_{x} b,(a, b) \mapsto a \wedge_{x} b,(a, b) \mapsto a \odot_{x} b,(a, b) \mapsto a \rightarrow_{x} b$ from the set $\{(a, b) \in F \times F \mid p(a)=p(b)\}$ into $F$ are continuous, where $x=p(a)=p(b)$;

(v) the functions $\underline{0}, \underline{1}: X \rightarrow F$, which assign to each $x$ in $X$ the zero $0_{x}$ and the unit $1_{x}$ of $F_{x}$ respectively, are continuous.

$X$ is known as the base space, $F$ as the total space and $F_{x}$ is called the stalk of $F$ at $x \in X$.

If $Y \subseteq X$, then a section $\sigma$ over $Y$ is a continuous map $\sigma: Y \rightarrow F$ satisfying $(p \circ \sigma)(y)=y$ for all $y \in Y$. The set of all sections over $Y$ form a nontrivial BLalgebra with the operations defined pointwise, that will be denoted by $\Gamma(Y, F)$. The elements of $\Gamma(X, F)$ are called global sections.

For every $\sigma, \tau \in \Gamma(Y, F)$, we shall use the following notation: $[\sigma=\tau]=\{y \in Y \mid \sigma(y)=\tau(y)\}$.

A BL-sheaf space $(F, p, X)$ is called local if for each $x \in X$ the stalk $F_{x}$ is a local BL-algebra. 
We shall use the expression a BL-algebra of global sections to refer to any BLsubalgebra of $\Gamma(X, F)$. If $A$ is a BL-algebra of global sections, then for each $x \in X$, we define $p_{x}^{A}: A \rightarrow F_{x}$ by $p_{x}^{A}(\sigma)=\sigma(x)$ for all $\sigma \in A$. If $A=\Gamma(X, F)$, then we shall denote $p_{x}^{A}$ by $p_{x}$.

The following properties are well-known and will be used in the sequel. For details see $[20,3,19]$.

Proposition 2.1 Let $(F, p, X)$ be a BL-sheaf space.

(i) for any $Y \subseteq X$ and $\sigma, \tau \in \Gamma(Y, F)$, the subset $[\sigma=\tau]$ is open in $Y$;

(ii) for each $a \in F$ there are an open subset $U$ of $X$ and a section $\sigma \in \Gamma(U, F)$ such that $p(a) \in U$ and $\sigma(p(a))=a$;

(iii) if $Z \subseteq Y \subseteq X$ and $\sigma \in \Gamma(Y, F)$, then $\left.\sigma\right|_{Z} \in \Gamma(Z, F)$;

(iv) the family $\{\sigma(U) \mid U$ is open in $X, \sigma \in \Gamma(U, F)\}$ is a basis for the topology of $F$;

(v) if $A$ is a BL-algebra of global sections, then $p_{x}^{A}$ is a BL-morphism for each $x \in X$;

(vi) if $(F, p, X)$ and $(G, q, X)$ are BL-sheaf spaces and $f: F \rightarrow G$ such that $q \circ f=p$, then

$f$ is continuous iff $f$ is open iff $f$ is a local homeomorphism.

If $A$ is a BL-algebra of global sections, $U$ is an open subset of $X$ and $\sigma$ is a section over $U$, we say that $\sigma$ is locally in the BL-algebra of global sections $A$ if $\left(^{*}\right)$ there are an open covering $\left(U_{i}\right)_{i \in I}$ of $U$ and a family $\left(\sigma_{i}\right)_{i \in I}$ of elements of $A$ such that $\left.\sigma\right|_{U_{i}}=\left.\sigma_{i}\right|_{U_{i}}$ for all $i \in I$.

The following lemma follows immediately from Proposition 2.1(iv).

Lemma 2.2 Let $(F, p, X)$ be a BL-sheaf space and $A$ a BL-algebra of global sections such that every section over an open subset of $X$ is locally in the BLalgebra $A$. Then the family $\{\sigma(U) \mid U$ is open in $X, \sigma \in A\}$ is a basis for the topology of $F$.

Proposition 2.3 Let $(F, p, X)$ be a BL-sheaf space and $A$ a BL-algebra of global sections. The following are equivalent:

(i) every section over an open subset of $X$ is locally in the BL-algebra $A$;

(ii) for each $x \in X$, the BL-morphism $p_{x}^{A}$ is onto.

Proof: (i) $\Rightarrow$ (ii) Let $x \in X$ and $a \in F_{x}$, that is $a \in F$ such that $p(a)=x$. Applying Proposition 2.1(ii), there is an open neighbourhood $U$ of $x$ and a section $\sigma$ over $U$ such that $\sigma(x)=a$. By (i), we get an open covering $\left(U_{i}\right)_{i \in I}$ of $U$ and a family $\left(\sigma_{i}\right)_{i \in I}$ of sections from $A$ such that $\left.\sigma\right|_{U_{i}}=\sigma-\left.i\right|_{U_{i}}$ for all $i \in I$. Since $x \in U$, we have that $x \in U_{k}$ for some $k \in I$. It follows that $\sigma_{k}(x)=\sigma(x)=a$. Hence, we have got $\sigma_{k} \in A$ such that $p_{x}^{A}\left(\sigma_{k}\right)=a$. That is, $p_{x}^{A}$ is onto.

(ii) $\Rightarrow$ (i) Let $U$ be an open subset of $X$ and $\sigma$ a section over $U$. For each $x \in U$, 
we have that $\sigma(x) \in F_{x}$, hence, by (ii), there is $\tau^{x} \in A$ such that $\tau^{x}(x)=\sigma(x)$. Applying Proposition 2.1(iii) and (i), it follows that $\left.\tau^{x}\right|_{U} \in \Gamma(U, F)$ and the subset $U_{x}=\left[\left.\tau^{x}\right|_{U}=\sigma\right]$ is an open subset of $U$ such that $x \in U_{x}$. Thus, we have got an open covering $\left(U_{x}\right)_{x \in U}$ of $U$ and a family $\left(\tau^{x}\right)_{x \in U}$ of sections from $A$ such that $\left.\tau^{x}\right|_{U_{x}}=\left.\left(\left.\tau^{x}\right|_{U}\right)\right|_{U_{x}}=\left.\sigma\right|_{U_{x}}$ for all $x \in U$.

Let $(F, p, X)$ be a BL-sheaf space and $\sigma \in \Gamma(Y, F)$ a section over $Y \subseteq X$. The cosupport of $\sigma, \operatorname{cosupp}(\sigma)$, is the closed hull in the subspace $Y$ of the set of those points $x \in Y$ for which $\sigma(x) \neq 1_{x}$ :

$$
\operatorname{cosupp}(\sigma)=\overline{\left\{x \in Y \mid \sigma(x) \neq 1_{x}\right\}} \text {. }
$$

It is easy to see that $(\operatorname{cosupp}(\sigma))^{c}=\left[\sigma=\left.\underline{1}\right|_{Y}\right]$.

Let $X$ and $Y$ be topological spaces and $f: Y \rightarrow X$ a continuous function. Let $(F, p, X)$ and $(G, q, Y)$ be two BL-sheaf spaces. A morphism $\alpha: F \rightarrow G$ over $f$ is a family $\left(\alpha_{y}: F_{f(y)} \rightarrow G_{y}\right)_{y \in Y}$ of BL-morphisms satisfying the following condition:

If $U$ is open in $X$ and $\sigma \in \Gamma(U, F)$, define $\beta: f^{-1}(U) \rightarrow G$ by $\beta(y)=$ $\alpha_{y}(\sigma(f(y)))$. Then $\beta$ is continuous, and therefore $\beta \in \Gamma\left(f^{-1}(U), G\right)$. We shall write $\beta=\alpha_{\#}^{U}(\sigma)$.

It follows that a morphism $\alpha: F \rightarrow G$ over $f$ induces a BL-morphism $\alpha_{\#}^{U}$ : $\Gamma(U, F) \rightarrow \Gamma\left(f^{-1}(U), G\right)$ for all open $U$ in $X$. We shall denote $\alpha_{\#}^{X}$ by $\varphi_{\#}$. Since $f^{-1}(X)=Y, \alpha_{\#}$ is a BL-morphism between the BL-algebras of global sections $\Gamma(X, F)$ and $\Gamma(Y, G)$.

An example of a morphism over $f$ is given by the canonical mapping from a BL-sheaf space $(F, p, X)$ to the BL-sheaf space $\left(f^{-1}(F), q, Y\right)$, induced by $f$ and $(F, p, X)$, defined as follows.

Define $f^{-1}(F)=\{(y, a) \in Y \times F \mid f(y)=p(a)\}=\bigcup_{y \in Y}\{y\} \times F_{f(y)}$ and $q: f^{-1}(F) \rightarrow Y$ by $q(y, a)=y$. Then for all $y \in Y, f^{-1}(F)_{y}=\{y\} \times F_{f(y)}$. For each $y \in Y$, define $i_{y}: F_{f(y)} \rightarrow f^{-1}(F)_{y}$ by $i_{y}(a)=(y, a)$. We get easily that $i_{y}$ is a bijection. We make $f^{-1}(F)_{y}$ a BL-algebra by transporting the BL-structure of $F_{f(y)}$ to $f^{-1}(F)_{y}$ by means of $i_{y}$.

Thus, we have got a BL-sheaf space $\left(f^{-1}(F), q, Y\right)$ and a morphism $i: F \rightarrow$ $f^{-1}(F)$ over $f$, where $i$ is the family $\left(i_{y}\right)_{y \in Y \text {. }}$

A morphism of BL-sheaf spaces $(f, \alpha):(F, p, X) \rightarrow(G, q, Y)$ consists of a continuous function $f: Y \rightarrow X$ and a morphism $\alpha: F \rightarrow G$ over $f$.

An isomorphism of BL-sheaf spaces is a morphism $(f, \alpha)$ such that $f$ is a homeomorphism and $\alpha_{y}$ is an isomorphism of BL-algebras for all $y \in Y$.

If $(f, \alpha):(F, p, X) \rightarrow(G, q, Y)$ and $(g, \beta):(G, q, y) \rightarrow(H, r, Z)$ are two morphisms of Bl-sheaf spaces, then their composition is the morphism $(f \circ g, \beta \circ \alpha)$, where $(\beta \circ \alpha)_{z}=\beta_{z} \circ \alpha_{g(z)}$ for all $z \in Z$.

Let $(F, p, X)$ and $(G, q, X)$ be BL-sheaf spaces over the same topological space $X$. If $\left(\alpha_{x}: F_{x} \rightarrow G_{x}\right)_{x \in X}$ is a family of functions, then we can define a function $\alpha: F \rightarrow G$ by $\alpha(a)=\alpha_{x}(a)$, where $x \in X$ is unique such that $a \in F_{x}$. 
Conversely, a function $\alpha: F \rightarrow G$ can be seen as a family $\left(\alpha_{x}: F_{x} \rightarrow G_{x}\right)_{x \in X}$, where $\alpha_{x}=\alpha \mid F_{x}$ for all $x \in X$.

Proposition $2.4\left(1_{X}, \alpha\right):(F, p, X) \rightarrow(G, q, X)$ is a morphism of BL-sheaf spaces iff $\alpha: F \rightarrow G$ is a continuous function such that $q \circ \alpha=p$ and $\alpha_{x}: F_{x} \rightarrow$ $G_{x}$ is a BL-morphism for all $x \in X$.

Let us denote by $B L$ the category of nontrivial BL-algebras and BL-morphisms and by $B L-S h S p$ the category of BL-sheaf spaces and morphisms of BL-sheaf spaces.

Define $\mathcal{S}(F, p, X)=\Gamma(X, F)$ for any BL-sheaf space $(F, p, X)$ and $\mathcal{S}(f, \alpha)=\alpha_{\#}$ for every morphism $(f, \alpha):(F, p, X) \rightarrow(G, q, Y)$. Then

Proposition 2.5 $\mathcal{S}: B L-S h S p \rightarrow B L$ is a functor, called the section functor.

\section{Compact BL-sheaf spaces}

Throughout, BL-algebras are nontrivial and $X$ will be assumed to denote a Hausdorff topological space.

A BL-sheaf space $(F, p, X)$ is called completely regular if it satisfies the following: (CR) for each $x \in X$ and closed set $C \subseteq X$ not containing $x$, there is $\sigma \in$ $\Gamma(X, F)$ such that $\sigma(x)=0_{x}$ and $\left.\sigma\right|_{C}=\underline{1} \mid C$.

A completely regular BL-sheaf space $(F, p, X)$ is called compact if the topological space $X$ is compact.

The following lemma gives equivalent characterizations of completely regular BL-sheaf spaces.

Lemma 3.1 Let $(F, p, X)$ be a BL-sheaf space. The following are equivalent:

(i) $(F, p, X)$ is completely regular;

(ii) for each $x \in X$ and every open neighbourhood $U$ of $x$ there is $\sigma \in \Gamma(X, F)$ such that $\sigma(x)=0_{x}$ and $\sigma(y)=1_{y}$ for all $y \notin U$;

(iii) for each $x \in X$ and every open neighbourhood $U$ of $x$ there is $\sigma \in \Gamma(X, F)$ such that $\sigma(x)=0_{x}$ and $\operatorname{cosupp}(\sigma) \subseteq U$.

Proof: (i) $\Rightarrow$ (ii) Let $C=U^{c}$. Then $C$ is a closed subset of $X$ such that $x \notin C$, and applying (i) we get (ii).

(ii) $\Rightarrow$ (i) Take $U=C^{c}$ and apply (ii).

(ii) $\Leftrightarrow$ (iii) Apply the fact that $(\operatorname{cosupp}(\sigma))^{c}=[\sigma=\underline{1}]$.

Proposition 3.2 Let $(F, p, X)$ be a completely regular BL-sheaf space. Then

(i) $X$ is a regular topological space;

(ii) every section over an open subset of $X$ is locally in the BL-algebra $\Gamma(X, F)$

of global sections of the BL-sheaf space;

(iii) the family $[\sigma=\underline{1}]_{\sigma \in \Gamma(X, F)}$ form a basis for the topology of $X$;

(iv) $F_{x} \cong A / \operatorname{Ker}\left(p_{x}\right)$ for all $x \in X$. 
Proof: (i) Let $x \in X$ and $U$ an open neighbourhood of $x$. Applying Lemma 3.1(iii), there is $\sigma \in \Gamma(X, F)$ such that $\sigma(x)=0_{x}$ and $\operatorname{cosupp}(\sigma) \subseteq U$. Hence, $x \in[\sigma=\underline{0}]$ and, since $F_{y}$ is nontrivial for all $y \in X$, we have that $0_{y} \neq 1_{y}$ for all $y \in X$, so $x \in[\sigma=\underline{0}] \subseteq \operatorname{cosupp}(\sigma)$. Hence, there is a closed neighbourhood $C=\operatorname{cosupp}(\sigma)$ of $x$ such that $C \subseteq U$. Thus, the closed neighbourhoods of $x$ form a basis for neighbourhoods, so $X$ is regular.

(ii) We shall prove that there is satisfied (ii) from Proposition 2.3 with $A=$ $\Gamma(X, F)$. Hence, we have to show that for each $x \in X, p_{x}$ is onto. Let $a \in F_{x}$, that is $a \in F$ such that $p(a)=x$. Applying Proposition 2.1(ii), there is an open neighbourhood $U$ of $x$ and a section $\tau$ over $U$ such that $\tau(x)=a$. By Lemma 3.1(iii), there is $\theta \in \Gamma(X, F)$ such that $\theta(x)=0_{x}$ and $\operatorname{cosupp}(\theta) \subseteq U$. Let $\sigma: X \rightarrow F$ defined by $\sigma(y)=\theta(y)^{-} \rightarrow_{y} \tau(y)$ for $y \in U$ and $\sigma(y)=1_{y}$ for $y \notin U$. It is obvious that $p \circ \sigma=1_{X}$ and that $p_{x}(\sigma)=\sigma(x)=\theta(x)^{-} \rightarrow_{x}$ $\tau(x)=0_{x}^{-} \rightarrow_{x} a=1_{x} \rightarrow a=a$. It remains to prove that $\sigma$ is continuous. Since $\operatorname{cosupp}(\theta) \subseteq U$, we get that $U \cup(\operatorname{cosupp}(\theta))^{c}=X$. Let us prove that $\sigma(y)=1_{y}$ for all $y \in(\operatorname{cosupp}(\theta))^{c}$. If $y \notin U$, then $\sigma(y)=1_{y}$ by the definition of $\sigma$. If $y \in U \cap(\operatorname{cosupp}(\theta))^{c}$, then $\theta(y)=1_{y}$ and $\sigma(y)=\theta(y)^{-} \rightarrow_{y} \tau(y)=$ $1_{y}^{-} \rightarrow_{y} \tau(y)=0_{y} \rightarrow \tau(y)=1_{y}$. Hence, we have got that $\left.\left.\sigma\right|_{U},\left.\sigma\right|_{(} \operatorname{cosupp}(\theta)\right)^{c}$ are continuous and $U,(\operatorname{cosupp}(\theta))^{c}$ form an open covering of $X$. It follows that $\sigma$ is continuous. Thus, we have obtained $\sigma \in \Gamma(X, F)$ such that $p_{x}(\sigma)=a$.

(iii) We have that $[\sigma=\underline{1}]$ is open in $X$ for all $\sigma \in \Gamma(X, F)$. We shall prove that for any $x \in X$ and any open neigbourhood $U$ of $x$ there is $\sigma \in \Gamma(X, F)$ such that $x \in[\sigma=\underline{1}] \subseteq U$. From this we get immediately that $[\sigma=\underline{1}]_{\sigma \in \Gamma(X, F)}$ form a basis for the topology of $X$. Applying Lemma 3.1(iii), there is $\tau \in \Gamma(X, F)$ such that $\tau(x)=0_{x}$ and $\operatorname{cosupp}(\tau) \subseteq U$. Let $\sigma=\tau^{-}$. Then, $\sigma(x)=(\tau(x))^{-}=$ $0_{x}^{-}=1_{x}$, hence $x \in[\sigma=\underline{1}]$. If $y \in[\sigma=\underline{1}]$, then $\sigma(y)=1_{y}$, that is $(\tau(y))^{-}=1_{y}$. It follows that $\tau(y) \neq 1_{y}$, since $0_{y} \neq 1_{y}$, so $y \in \operatorname{cosupp}(\tau) \subseteq U$. Hence, we have proved that $[\sigma=\underline{1}]$ is an open neighbourhood of $x$ contained in $U$.

(iv) We have proved at (ii) that the BL-morphism $p_{x}: A \rightarrow F_{x}, p_{x}(\sigma)=\sigma(x)$ is onto. Hence, $F_{x} \cong A / \operatorname{Ker}\left(p_{x}\right)$.

Let $A$ be a BL-algebra of global sections of the BL-sheaf space $(F, p, X)$. We say that $A$ is completely regular in the $B L$-sheaf space $(F, p, X)$ if for each $x \in X$ and closed set $C \subseteq X$ not containing $x$, there is $\sigma \in A$ such that $\sigma(x)=0_{x}$ and $\left.\sigma\right|_{C}=\left.\underline{1}\right|_{C}$.

If $A$ is completely regular in $(F, p, X)$ and $X$ is compact, then $A$ is said to be compact in the $B L$-sheaf space $(F, p, X)$.

It is easy to see that, as in Lemma $3.1, A$ is completely regular in the BL-sheaf space $(F, p, X)$ iff for each $x \in X$ and every open neighbourhood $U$ of $x$ there is $\sigma \in A$ such that $\sigma(x)=0_{x}$ and $\sigma(y)=1_{y}$ for all $y \notin U$. The following result extends Proposition 3.2(i) and (iii) and its proof is similar.

Lemma 3.3 Let $A$ be a BL-algebra of global sections that is completely regular in $(F, p, X)$. Then

(i) $X$ is regular; 
(ii) the family $[\sigma=\underline{1}]_{\sigma \in A}$ form a basis for the topology of $X$.

The following lemma collects some obvious facts that will be used in the sequel.

Lemma 3.4 Let $(F, p, X)$ be a BL-sheaf space.

(i) $(F, p, X)$ is completely regular (compact) iff the BL-algebra $\Gamma(X, F)$ of global sections is completely regular (compact) in $(F, p, X)$;

(ii) Suppose that $A$ and $B$ are BL-algebras of global sections such that $A \subseteq B$. If $A$ is completely regular (compact) in $(F, p, X)$, then $B$ is completely regular (compact) in $(F, p, X)$;

(iii) If there is a BL-algebra $A$ of global sections that is completely regular (compact) in $(F, p, X)$, then $(F, p, X)$ is completely regular (compact).

Proposition 3.5 Let $A$ be a BL-algebra of global sections that is compact in $(F, p, X)$ and suppose that every global section is locally in $A$. Then $A$ is necessarily the BL-algebra $\Gamma(X, F)$.

Proof: Let $\sigma \in \Gamma(X, F)$. Since $\sigma$ is locally in $A$, it follows that there are an open covering $\left(U_{i}\right)_{i \in I}$ of $X$ and a family $\left(\sigma_{i}\right)_{i \in I}$ of elements of $A$ such that $\left.\sigma\right|_{U_{i}}=\left.\sigma_{i}\right|_{U_{i}}$ for all $i \in I$. For each $x \in X$, there is $i_{x} \in I$ such that $x \in U_{i_{x}}$ and applying the fact that $A$ is completely regular in $(F, p, X)$, we get $\tau_{i_{x}} \in A$ such that $\tau_{i_{x}}(x)=0_{x}$ and $\tau_{i_{x}}(y)=1_{y}$ for all $y \notin U_{i_{x}}$. Let us denote $U_{i_{x}} \cap\left[\tau_{i_{x}}=\underline{0}\right]$ by $V_{x}$. Then, $x \in V_{x} \subseteq U_{i_{x}}$ for all $x \in X$, so the family $\left(V_{x}\right)_{x \in X}$ is an open covering of $X$. Since $X$ is compact, it follows that there are $x_{1}, \cdots, x_{n} \in X$ such that $X=V_{x_{1}} \cup \cdots \cup V_{x_{n}}$. Let us denote $V_{x_{k}}$ by $V_{k}, i_{x_{k}}$ by $i_{k}$ and $\tau_{i_{x_{k}}}$ by $\tau_{k}$ for all $k=\overline{1, n}$. We shall prove that $\sigma=\bigwedge_{k=\overline{1, n}}\left(\sigma_{i_{k}} \vee \tau_{k}\right)$. Let $x \in X$ and $J=\left\{k=\overline{1, n} \mid x \in U_{i_{k}}\right\}$. It is obvious that $J$ is nonempty, since $\bigcup_{k=1, n} U_{i_{k}}=$ $X$. We have that $\sigma_{i_{k}}(x)=\sigma(x)$ for all $k \in J$ and $x \notin U_{i_{k}}$ for all $k \notin J$, so $\tau_{k}(x)=1_{x}$ for all $k \notin J$. It follows that $\left[\bigwedge_{k \in J}\left(\sigma_{i_{k}}(x) \vee \tau_{k}(x)\right)\right] \wedge\left[\bigwedge_{k \notin J}\left(\sigma_{i_{k}}(x) \vee\right.\right.$ $\left.\left.\tau_{k}(x)\right)\right]=\left[\bigwedge_{k \in J}\left(\sigma(x) \vee \tau_{k}(x)\right)\right] \wedge\left[\bigwedge_{k \notin J}\left(\sigma_{i_{k}}(x) \vee 1_{x}\right]=\sigma(x) \vee \bigwedge_{k \in J} \tau_{k}(x)\right.$. Since $X=\bigcup_{k=1, n} V_{k}$, there is $j=\overline{1, n}$ such that $x \in V_{j}$, so $\tau_{j}(x)=0_{x}$ and $j \in J$, since $V_{j} \subseteq U_{i_{j}}$. It follows that $\left(\bigwedge_{k \in J} \tau_{k}\right)(x)=0_{x}$, hence $\left[\bigwedge_{k=\overline{1, n}}\left(\sigma_{i_{k}} \vee \tau_{k}\right)\right](x)=\sigma(x)$. Thus, $\sigma=\bigwedge_{k=\overline{1, n}}\left(\sigma_{i_{k}} \vee \tau_{k}\right)$, hence $\sigma \in A$.

\subsection{The compactness theorem}

In the sequel, $A$ will be a BL-algebra of global sections of the BL-sheaf space $(F, p, X)$.

For each $x \in X$, let us denote $K_{x}=\operatorname{Ker}\left(p_{x}^{A}\right)=\left\{\sigma \in A \mid \sigma(x)=1_{x}\right\}$. Since $A$ is nontrivial, it follows that $K_{x}$ is a proper filter of $A$.

A filter $T$ of $A$ is called fixed if there is $x \in X$ such that $T \vee K_{x}$ is a proper filter of $A$. Otherwise, $T$ is said to be a free filter of $A$. 
Lemma 3.6 Let $A$ be a BL-algebra of global sections of $(F, p, X), P$ a prime filter and $M$ a maximal filter of $A$. Then

(i) $M$ is fixed iff $M$ contains the filter $K_{x}$ for some $x \in X$;

(ii) if $M_{P}$ is the unique maximal filter that contains $P$, then $P$ is fixed iff $M_{P}$ is fixed;

(iii) if $P$ contains the filter $K_{x}$ for some $x \in X$, then $P$ is fixed.

Proof: (i) Suppose that $M$ is fixed, so there is $x \in X$ such that $M \vee K_{x}$ is a proper filter of $A$. Since $M \subseteq M \vee K_{x}$ and $M$ is maximal, it follows that $M \vee K_{x}=M$, hence $K_{x} \subseteq M$. Conversely, if $K_{x} \subseteq M$ for some $x \in X$, we get that $M \vee K_{x}=M$, so $M \vee K_{x}$ is a proper filter of $A$. That is, $M$ is fixed.

(ii) If $M_{P}$ is fixed, then, by (i), there is $x \in X$ such that $K_{x} \subseteq M_{P}$. Since $P \subseteq M_{P}$, we have that $P \vee K_{x} \subseteq M_{P}$, hence $P \vee K_{x}$ is a proper filter of $A$, i.e. $P$ is fixed. Conversely, suppose that $P$ is fixed, that is $P \vee K_{x}$ is proper for some $x \in X$. We get that $M_{P}$ and $P \vee K_{x}$ are proper filters containing the prime filter $P$, so applying Proposition 1.2 and the fact that $M_{P}$ is maximal, it follows that $P \vee K_{x} \subseteq M_{P}$. Hence, $K_{x} \subseteq M_{P}$, so by (i), $M_{P}$ is fixed.

(iii) Since $K_{x} \subseteq M_{P}$, we get that $M_{P}$ is fixed, by (i). Applying (ii), we obtain that $P$ is also fixed.

Lemma 3.7 Let $A$ be a BL-algebra of global sections of $(F, p, X)$. The following are equivalent

(i) every proper filter of $A$ is fixed;

(ii) every prime filter of $A$ is fixed;

(iii) every maximal filter of $A$ is fixed.

Proof: (i) $\Rightarrow$ (ii) Obviously.

(ii) $\Rightarrow$ (iii) Apply the fact that $\operatorname{Max}(A) \subseteq \operatorname{Spec}(A)$, by Proposition 1.4.

(iii) $\Rightarrow$ (i) Let $F$ be a proper filter of $A$. By Proposition 1.1, there is a maximal filter $M$ such that $F \subseteq M$. Since $M$ is fixed, we get $x \in X$ such that $K_{x} \subseteq M$. We have that $F, K_{x} \subseteq M$, so $F \vee K_{x} \subseteq M$. Hence, $F \vee K_{x}$ is a proper filter of $A$, that is $F$ is fixed.

Lemma 3.8 Let $A$ be a BL-algebra of global sections of $(F, p, X)$ and suppose that $X$ is compact. Then

(i) for every prime filter $P$ of $A$ there is $x \in X$ such that $K_{x} \subseteq P$;

(ii) every proper filter of $A$ is fixed.

Proof: (i) Let $P$ be a prime filter of $A$ and suppose that $K_{x} \nsubseteq P$ for any $x \in X$. That is for any $x \in X$ there is $\sigma^{x} \in K_{x}$ such that $\sigma^{x} \notin P$. Since $\sigma^{x} \in K_{x}$, we get that $\sigma^{x}(x)=1_{x}$, that is $x \in\left[\sigma^{x}=\underline{1}\right]$. Thus, $X=\bigcup_{x \in X}\left[\sigma^{x}=\underline{1}\right]$, hence the family $\left[\sigma^{x}=\underline{1}\right]_{x \in X}$ is an open covering of $X$. Since $X$ is compact, it follows that there are $x_{1}, \cdots, x_{n} \in X$ such that $X=\bigcup_{i=1}^{n}\left[\sigma_{i}=\underline{1}\right]$, where $\sigma_{i}$ denotes $\sigma^{x_{i}}$ for $i=\overline{1, n}$. It follows immediately that $\sigma_{1} \vee \cdots \vee \sigma_{n}=\underline{1} \in P$. Since $P$ is prime, we obtain that $\sigma_{i} \in P$ for some $i=\overline{1, n}$. Thus, we have got a contradiction. 
(ii) Applying (i) and Lemma 3.6(iii), we obtain that every prime filter of $A$ is fixed. Now apply Lemma 3.7 to get that every proper filter of $A$ is fixed.

In the following, we shall denote by $\operatorname{Spec}_{X}(A)$ the set of prime filters of $A$ that are fixed and by $\operatorname{Max}_{X}(A)$ the set of maximal filters of $A$ that are fixed.

Lemma 3.9 Suppose that $A$ is completely regular in $(F, p, X)$. Then

(i) for any $P \in \operatorname{Spec}_{X}(A)$ there is a unique $x \in X$ such that $K_{x} \subseteq M_{P}$, where $M_{P}$ is the unique maximal filter that contains $P$;

(ii) for any $M \in \operatorname{Max}_{X}(A)$ there is a unique $x \in X$ such that $K_{x} \subseteq M$.

Proof: (i) The existence of $x \in X$ such that $K_{x} \subseteq M_{P}$ follows from Lemma 3.6. It remains to prove the unicity. Let us suppose that there is $y \neq x$ such that $K_{y} \subseteq M_{P}$. Since $X$ is Hausdorff, there is an open neighbourhood $U$ of $x$ such that $y \notin U$. Applying now Lemma 3.1(ii), there is $\sigma \in A$ such that $\sigma(x)=0_{x}$ and $\sigma(z)=1_{z}$ for all $z \notin U$. It follows that $\sigma(y)=1_{y}$, so $\sigma \in K_{y} \subseteq M_{P}$ and $\sigma^{-}(x)=1_{x}$, hence $\sigma^{-} \in K_{x} \subseteq M_{P}$. We have got that $\sigma, \sigma^{-} \in M_{P}$, hence $\sigma \odot \sigma^{-}=\underline{0} \in M_{P}$. Thus, we have obtained that $M_{P}$ is not proper, that is a contradiction.

(ii) By (i).

If $A$ is completely regular in $(F, p, X)$, then, by the above lemma, we can define a function $\mathbf{s}: \operatorname{Spec}_{X}(A) \rightarrow X$ that assigns to each $P \in \operatorname{Spec}_{X}(A)$ the unique $x \in X$ such that $K_{x} \subseteq M_{P}$. We shall denote by $\mathbf{m}$ its restriction to $\operatorname{Max}_{X}(A)$. Then $\mathbf{m}$ assigns to every fixed maximal filter $M$ of $A$ the unique $x \in X$ such that $K_{x} \subseteq M$.

Corollary 3.10 Let $A$ be a BL-algebra of global sections of $(F, p, X)$ and suppose that $X$ is compact. Then for every prime filter $P$ of $A$ there is a unique $x \in X$ such that $K_{x} \subseteq P$.

Proof: Apply Lemmas 3.8 and 3.9.

Lemma 3.11 Suppose that $A$ is completely regular in $(F, p, X)$. Then for any $M \in \operatorname{Max}_{X}(A), K_{\mathbf{m}(M)} \subseteq O(M)$

Proof: Let $x=\mathbf{m}(M)$ and $\sigma \in K_{x}$. We get that $\sigma(x)=1_{x}$, so $x \in[\sigma=\underline{1}$. Applying the fact that $A$ is completely regular in $(F, p, X)$, we get $\tau \in A$ such that $\tau(x)=0_{x}$ and $\tau(y)=1_{y}$ for all $y \notin[\sigma=\underline{1}]$. It is clear that $\sigma \vee \tau=\underline{1}$. ¿From $\tau(x)=0_{x}$, it follows that $\tau^{-}(x)=1_{x}$, so $\tau^{-} \in K_{x} \subseteq M$. Since $M$ is proper, we must have $\tau \notin M$. Hence, there is $\tau \notin M$ such that $\sigma \vee \tau=\underline{1}$, that is $\sigma \in O(M)$.

Lemma 3.12 Let $(F, p, X)$ be a completely regular local BL-sheaf space and $A=\Gamma(X, F)$. Then

(i) for any $x \in X$ there is a unique $M \in \operatorname{Max}(A)$ such that $K_{x} \subseteq M$;

(ii) $K_{\mathbf{m}(M)}=O(M)$ for any $M \in \operatorname{Max}_{X}(A)$. 
Proof: (i) By Proposition 3.2(v) and the fact that $\operatorname{Ker}\left(p_{x}\right)=K_{x}$, it follows that $F_{x} \cong A / K_{x}$ for all $x \in X$. Hence, $A / K_{x}$ is local for any $x \in X$. Apply now Proposition 1.13.

(ii) Applying Proposition 3.11, we have that $K_{\mathbf{m}(M)} \subseteq O(M)$. Let us prove the converse inclusion. If we denote $x=\mathbf{m}(M)$, then $K_{x} \subseteq M$. Let $\sigma \in O(M)$, so there is $\tau \notin M$ such that $\sigma \vee \tau=\underline{1}$. Since $F_{x}$ is local, its unique maximal filter is $N_{x}=\left\{a \in F_{x} \mid \operatorname{ord}(a)=\infty\right\}$. By Proposition 1.10, we have that $p_{x}^{-1}\left(N_{x}\right)$ is a maximal filter of $A$ and it is easy to see that $K_{x} \subseteq p_{x}^{-1}\left(N_{x}\right)$. Since $K_{x} \subseteq p_{x}^{-1}\left(N_{x}\right), K_{x} \subseteq M$ and $p_{x}^{-1}\left(N_{x}\right), M$ are maximal filters of $A$, applying (i) it follows that $p_{x}^{-1}\left(N_{x}\right)=M$. Now, $\tau \notin M$ implies $\tau \notin p_{x}^{-1}\left(N_{x}\right)$, so $\left.\operatorname{ord}(\tau(x))\right)<$ $\infty$. Thus, there is $n \in \omega-\{0\}$ such that $(\tau(x))^{n}=0_{x}$. Since $\sigma \vee \tau=\underline{1}$, we get that $\sigma(x) \vee_{x} \tau(x)=1_{x}$, so $(\sigma(x))^{n} \vee_{x}(\tau(x))^{n}=1_{x}$, that is $(\sigma(x))^{n}=1_{x}$, hence $\sigma(x)=1_{x}$. Thus, we have got that $\sigma \in K_{x}$.

Proposition 3.13 Let $A$ be completely regular in $(F, p, X)$. Then $\mathbf{s}$ is onto and $\mathbf{m}$ is continuous and onto.

Proof: Let $x \in X$. Then $K_{x}$ is a proper filter of $A$, so, by Proposition 1.1, there is a maximal filter $M$ such that $K_{x} \subseteq M$. Applying Lemma 3.6(i), we get that $M$ is fixed. Hence, $M \in \operatorname{Max}_{X}(A)$ is such that $\mathbf{m}(M)=x$. Thus, $\mathbf{m}$ is onto and, obviously, $\mathbf{s}$ is also onto. Let us prove now that $\mathbf{m}$ is continuous. Let $M \in \operatorname{Max}_{X}(A), x=\mathbf{m}(M)$ and $U$ an open neighbourhood of $x$. Since $A$ is completely regular in $(F, p, X))$, there is $\sigma \in A$ such that $\sigma(x)=0_{x}$ and $\sigma(y)=$ $1_{y}$ for all $y \notin U$. Let $V=d(\sigma) \cap \operatorname{Max}_{X}(A)=\left\{N \in \operatorname{Max}_{X}(A) \mid \sigma \notin N\right\}$. Then $V$ is an open subset of $\operatorname{Max}_{X}(A)$. Since $\sigma(x)=0_{x}$, we get that $\sigma^{-}(x)=1_{x}$, that is $\sigma^{-} \in K_{x} \subseteq M$. It follows that $\sigma \notin M$, hence $M \in V$. Let us prove that $\mathbf{m}(V) \subseteq U$. Let $N \in V$ and $y=\mathbf{m}(N)$, so $K_{y} \subseteq N$. If $y \notin U$, then $\sigma(y)=1_{y}$, so $\sigma \in K_{y}$, hence $\sigma \in N$. This contradicts the fact that $N \in d(\sigma)$. It follows that $y \in U$. Thus, we have proved that $V$ is an open neighbourhood of $M$ such that $\mathbf{m}(V) \subseteq U$. That is, $\mathbf{m}$ is continuous at $M$.

Suppose that $A$ is compact in $(F, p, X)$. Then, by Lemma 3.8, we have that $\operatorname{Spec}_{X}(A)=\operatorname{Spec}(A)$ and, by Corollary $3.10, \mathbf{s}: \operatorname{Spec}(A) \rightarrow X$ assigns to every prime filter $P$ of $A$ the unique $x \in X$ such that $K_{x} \subseteq P$. We obtain the following corollary.

Corollary 3.14 Let $A$ be compact in the BL-sheaf space $(F, p, X)$. Then $\mathbf{s}$ and $\mathbf{m}$ are continuous, closed and onto.

Proof: We get that $\mathbf{s}$ is continuous in a similar manner with the proof of continuity of $\mathbf{m}$ from Proposition 3.13. To obtain that the functions are closed, apply [17, Theorem 7.2.2, p. 71], since $\mathbf{s}, \mathbf{m}$ are continuous and onto, $\operatorname{Max}(A)$ and $\operatorname{Spec}(A)$ are compact and $X$ is Hausdorff.

Theorem 3.15 (The compactness theorem)

Suppose that $A$ is completely regular in the BL-sheaf space $(F, p, X)$. The 
following are equivalent

(i) the topological space $X$ is compact;

(ii) every proper filter of $A$ is fixed;

(iii) every maximal filter of $A$ is fixed;

(iv) every prime filter of $A$ is fixed;

(v) $A$ is compact in the BL-sheaf space $(F, p, X)$.

Proof: (i) $\Leftrightarrow(\mathrm{v})$ By definition.

(ii) $\Leftrightarrow$ (iii) $\Leftrightarrow$ (iv) By Lemma 3.7.

(i) $\Rightarrow$ (ii) Apply Lemma 3.8.

(ii) $\Rightarrow$ (i) We have that $\operatorname{Max}_{X}(A)=\operatorname{Max}(A)$ and $\mathbf{m}: \operatorname{Max}(A) \rightarrow X$. Since $\mathbf{m}$ is continuous and onto and $\operatorname{Max}(A)$ is compact, applying a known result of topology, it follows that $X$ is also compact (see, e.g., [17, Theorem 7.2.1, p.71]).

Proposition 3.16 If $(F, p, X)$ is a compact BL-sheaf space and $A=\Gamma(X, F)$, then

$\mathbf{m}$ is a homeomorphism iff $(F, p, X)$ is a local BL-sheaf space.

Proof: Applying Propositions 1.13 and 3.2(iv), it follows that $\mathbf{m}$ is injective iff for any $x \in X$ there is a unique maximal filter $M$ of $A$ such that $\mathbf{m}(M)=x$ iff for any $x \in X$ there is a unique maximal filter $M$ of $A$ such that $K_{x} \subseteq M$ iff for all $x \in X, A / K_{x}$ is local iff for all $x \in X, F_{x}$ is a local BL-algebra. Hence, if $\mathbf{m}$ is a homeomorphism, then $(F, p, X)$ is a local BL-sheaf space. Conversely, if $(F, p, X)$ is local, then $\mathbf{m}$ is injective. We have that $\mathbf{m}$ is bijective, continuous and closed, by Corollary 3.14. Hence, $\mathbf{m}$ is a homeomorphism.

Let $(F, p, X)$ be a compact local BL-sheaf space and $A=\Gamma(X, F)$. By the proof of the above proposition, we can define a function $\mathbf{n}: X \rightarrow \operatorname{Max}(A)$, that associates with every $x \in X$ the unique maximal filter $M$ of $A$ such that $K_{x} \subseteq M$. It is easy to see that

Proposition 3.17 Let $(F, p, X)$ be a compact local BL-sheaf space. Then $\mathbf{n}$ is the inverse of $\mathbf{m}$, hence $\mathbf{n}: X \rightarrow \operatorname{Max}(A)$ is also a homeomorphism.

\section{Compact representations of BL-algebras}

In the sequel, $A$ will be a nontrivial BL-algebra and $X$ will be assumed to denote a Hausdorff topological space.

By a sheaf representation (or simply representation) of the BL-algebra $A$ will be meant a BL-morphism

$$
\varphi: A \rightarrow \Gamma(X, F)
$$

from $A$ to the BL-algebra of global sections of a BL-sheaf space $(F, p, X)$.

Hence, $\varphi(A)$ is a BL-algebra of global sections of $(F, p, X)$. In a representation 
$\varphi$, each $a \in A$ determines a global section $\varphi(a)$; in particular, for every $x \in X$, $\varphi(a)(x)$ is an element of the stalk $F_{x}$.

For each $x \in X$, we define

$$
\begin{aligned}
& \varphi_{x}: A \rightarrow F_{x}, \varphi_{x}(a)=\varphi(a)(x) \text { for all } a \in A, \\
& K_{x}=\operatorname{Ker}\left(\varphi_{x}\right)=\left\{a \in A \mid \varphi(a)(x)=1_{x}\right\} .
\end{aligned}
$$

Since $\varphi_{x}=p_{x} \circ \varphi$, we have that $\varphi_{x}$ is a BL-morphism, so $K_{x}$ is a proper filter of $A$ for every $x \in X$.

It is easy to see that $\operatorname{Ker}(\varphi)=\bigcap_{x \in X} K_{x}$, hence $\varphi$ is a monomorphism iff $\bigcap_{x \in X} K_{x}=\{1\}$.

For every $a \in A$, we shall use the following notation:

$$
V(a)=[\varphi(a)=\underline{1}]=\left\{x \in X \mid \varphi(a)(x)=1_{x}\right\}=\left\{x \in X \mid a \in K_{x}\right\} .
$$

By Proposition 2.1(i), $V(a)$ is open in $X$ for all $a \in A$.

A filter space of a BL-algebra $A$ is a family $\left(T_{x}\right)_{x \in X}$ of proper filters of $A$, indexed by a Hausdorff topological space $X$.

Let $\varphi: A \rightarrow \Gamma(X, F)$ be a representation of $A$. The filter space $\left(K_{x}\right)_{x \in X}$ will be called the representation space of the representation, and the filters indexed the representation filters. The topology generated by the family $(V(a))_{a \in A}$ of subsets of $X$ is called the representation topology on the space $X$. Then, any topology on $X$ contains the representation topology.

We say that a filter space $\left(T_{x}\right)_{x \in X}$ canonically determines a representation of $A$ if there is a representation $\varphi: A \rightarrow \Gamma(X, F)$ such that $T_{x}=K_{x}$ for all $x \in X$.

In the sequel, we shall give an existence theorem for representations of BLalgebras.

Let $A$ be a nontrivial BL-algebra and $\left(T_{x}\right)_{x \in X}$ a filter space of $A$ such that the subset $V(a)=\left\{x \in X \mid a \in T_{x}\right\}$ is open in $X$ for all $a \in A$. Then a BL-sheaf space $\left(F_{A}, p_{A}, X\right)$ and a representation $\varphi: A \rightarrow \Gamma\left(X, F_{A}\right)$ can be constructed in the following way, given in [3] for universal algebra. Let $F_{A}$ be the disjoint union of the sets $\left(A / T_{x}\right)_{x \in X}$ and $p_{A}: F_{A} \rightarrow X$ the canonical projection, so $p_{A}^{-1}(\{x\})=A / T_{x}$ for all $x \in X$. For all $x \in X, T_{x}$ is a proper filter of $A$, so $A / T_{x}$ is a nontrivial BL-algebra. For each $a \in A$, define the map $[a]: X \rightarrow F_{A}$ by $[a](x)=a / T_{x}$. Endow $F_{A}$ with the topology generated by the family $\{[a](U) \mid a \in A$ and $U$ is open in $X\}$. Applying [3, Corollary $2]$, we get that $\left(F_{A}, p_{A}, X\right)$ is a sheaf space of BL-algebras and the function $\varphi: A \rightarrow \Gamma\left(X, F_{A}\right)$, defined by $\varphi(a)=[a]$ for all $a \in A$, is a representation of $A$. It is easy to see that $K_{x}=T_{x}$ for all $x \in X$.

Hence, we get the following theorem:

Theorem 4.1 Let $A$ be a nontrivial BL-algebra and $\left(T_{x}\right)_{x \in X}$ a filter space of $A$ such that the subset $V(a)=\left\{x \in X \mid a \in T_{x}\right\}$ is open in $X$ for all $a \in A$. Then $\left(T_{x}\right)_{x \in X}$ canonically determines a representation of $A$.

In the sequel, we shall define completely regular and compact representations and, finally, we shall prove that any compact representation arises canonically from a filter space of the BL-algebra satisfying certain conditions. 
Thus, a representation $\varphi: A \rightarrow \Gamma(X, F)$ of a BL-algebra $A$ in a BL-sheaf space $(F, p, X)$ will be said to be a completely regular representation of $A$ if $\varphi$ is a monomorphism and $\varphi(A)$ is completely regular in $(F, p, X)$. A compact representation of $A$ is a monomorphism $\varphi: A \rightarrow \Gamma(X, F)$ such that $\varphi(A)$ is compact in $(F, p, X)$. Hence, a compact representation is a completely regular representation $\varphi: A \rightarrow \Gamma(X, F)$ with the property that $X$ is compact.

Proposition 4.2 Let $\varphi: A \rightarrow \Gamma(X, F)$ be a completely regular representation of $A$. Then

(i) for any distinct $x, y \in X$, there is $a \in A$ such that $\varphi(a)(x)=0_{x}$ and $\varphi(a)(y)=1_{y}$

(ii) the topology on $X$ is the representation topology.

Proof: (i) Since $X$ is Hausdorff, we have that $\{y\}$ is closed in $X$. Apply now the fact that $\varphi(A)$ is completely regular in $(F, p, X)$ for the closed set $\{y\}$ and $x \notin\{y\}$.

(ii) As we have noticed, the topology on $X$ contains the representation topology. For the converse, apply Proposition 3.3(ii).

For any BL-algebra $A$, a family $\left(T_{x}\right)_{x \in X}$ of proper filters of $A$ will be said to be coprime if $\bigcap_{x \in X} T_{x}=\{1\}$ and for any distinct $x, y \in X$ we have

$T_{x} \vee T_{y}=A$.

The family $\left(T_{x}\right)_{x \in X}$ is called strongly coprime if $\bigcap_{x \in X} T_{x}=\{1\}$ and for any $x \in X$ and $a \in T_{x}$, we have

$T_{x} \vee \bigcap\left\{T_{y} \mid y \in X\right.$ and $\left.a \notin T_{y}\right\}=A$.

In the sequel, let us consider a filter space $\left(T_{x}\right)_{x \in X}$ of $A$ such that the subset $V(a)=\left\{x \in X \mid a \in T_{x}\right\}$ is open in $X$ for all $a \in A$. By Theorem 4.1, there is a representation $\varphi: A \rightarrow \Gamma(X, F)$ of $A$ such that $T_{x}=K_{x}=\{a \in A \mid \varphi(a)(x)=$ $\left.1_{x}\right\}$ for all $x \in X$.

Lemma 4.3 If $\varphi$ is completely regular, then the family $\left(T_{x}\right)_{x \in X}$ is coprime.

Proof: Since $\varphi$ is a monomorphism, we have that $\bigcap_{x \in X} T_{x}=\{1\}$. Let $x, y \in X$ be two distinct points of $X$. Applying Proposition 4.2(ii), it follows that there is $a \in A$ such that $\varphi(a)(x)=0_{x}$ and $\varphi(a)(y)=1_{y}$. Hence, $a \in T_{y}$ and $a^{-} \in T_{x}$, since $\varphi\left(a^{-}\right)(x)=(\varphi(a)(x))^{-}=0_{x}^{-}=1_{x}$. We get that $0=a \odot a^{-} \in T_{x} \vee T_{y}$, i.e. $T_{x} \vee T_{y}=A$.

Proposition 4.4 The following are equivalent:

(i) $\varphi$ is completely regular;

(ii) the family $\left(T_{x}\right)_{x \in X}$ is strongly coprime and the topology on $X$ is generated by the family $V(a)_{a \in A}$.

Proof: $\quad(\mathrm{i}) \Rightarrow$ (ii) By Proposition 4.2(i), the topology on $X$ is generated by the family $V(a)_{a \in A}$. Let us prove that the family $\left(T_{x}\right)_{x \in X}$ is strongly coprime. Let 
$x \in X$ and $a \in T_{x}$. Since $\varphi(A)$ is completely regular in $(F, p, X)$ and $V(a)$ is an open neighbourhood of $x$, there is $b \in A$ such that $\varphi(b)(x)=0_{x}$ and $\varphi(b)(y)=1_{y}$ for all $y \notin V(a)$. It follows that $b^{-} \in T_{x}$ and $b \in \bigcap\left\{T_{y} \mid y \in X\right.$ and $\left.a \notin T_{y}\right\}$, so $0=b \odot b^{-} \in T_{x} \vee \bigcap\left\{T_{y} \mid y \in X\right.$ and $\left.a \notin T_{y}\right\}$.

(ii) $\Rightarrow$ (i) Let $x \in X$ and $U$ an open neighbourhood of $x$. Since the topology on $X$ is generated by the family $V(a)_{a \in A}$, there is $a \in A$ such that $x \in V(a) \subseteq U$. We have that $T_{x} \vee \bigcap\left\{T_{y} \mid y \in X\right.$ and $\left.a \notin T_{y}\right\}=A$, so there are $b \in T_{x}$ and $c \in \bigcap\left\{T_{y} \mid y \in X\right.$ and $\left.a \notin T_{y}\right\}$ such that $b \odot c=0$. Since $c \in T_{y}$ for all $y \in X$ such that $a \notin T_{y}$, we get that $\varphi(c)(y)=1_{y}$ for all $y \notin V(a)$, hence $\varphi(c)(y)=1_{y}$ for all $y \in U$, since $V(a) \subseteq U$. From $b \odot c=0$ we obtain that $b \leq c^{-}$, so $c^{-} \in T_{x}$, because $b \in T_{x}$ and $T_{x}$ is a filter of $A$. We get that $\varphi\left(c^{-}\right)(x)=1_{x}$, hence $\varphi(c)(x)=0_{x}$. Thus, for any $x \in X$ and any open neighbourhood $U$ of $x$ there is $c \in A$ such that $\varphi(c)(x)=0_{x}$ and $\varphi(c)(y)=1_{y}$ for all $y \notin U$. That is, $\varphi(A)$ is completely regular in $(F, p, X)$.

Theorem 4.5 Let $A$ be a nontrivial BL-algebra and $\left(T_{x}\right)_{x \in X}$ a filter space of $A$ such that the subset $V(a)=\left\{x \in X \mid a \in T_{x}\right\}$ is open in $X$ for all $a \in A$. The following are equivalent:

(i) the filter space canonically determines a compact representation of $A$;

(ii) $X$ is compact and the family $\left(T_{x}\right)_{x \in X}$ is coprime;

(iii) the family $\left(T_{x}\right)_{x \in X}$ is strongly coprime, the topology on $X$ is generated by the family $V(a)_{a \in A}$ and any maximal filter of $A$ contains a filter of the filter space.

Proof: (i) $\Rightarrow$ (ii) Obviously $X$ is compact. Apply Lemma 4.3 to get that $\left(T_{x}\right)_{x \in X}$ are coprime.

(ii) $\Rightarrow$ (i) Suppose that $U$ is an open subset of $X$ and let $x \in U$ and $C=U^{c}$. Then, for all $y \in C$ we have that $x \neq y$, so, by the fact that the family $\left(T_{x}\right)_{x \in X}$ is coprime, we obtain that $T_{x} \vee T_{y}=A$. Hence, for all $y \in C$, there are $a^{y} \in T_{x}$ and $b^{y} \in T_{y}$ such that $a^{y} \odot b^{y}=0_{y}$. It follows that $\left(b^{y}\right)^{-} \in T_{x}$ for all $y \in C$. We also get that $y \in V\left(b^{y}\right)$ for all $y \in C$, so $C \subseteq \bigcup_{y \in C} V\left(b^{y}\right)$. Since $C$ is a closed subset of the compact space $X, C$ is also compact, hence there are $y_{1}, \cdots y_{n} \in C$ and $b_{1}=b^{y_{1}}, \cdots b_{n}=b^{y_{n}} \in T_{y}$ such that $C \subseteq V\left(b_{1}\right) \cup \cdots \cup V\left(b_{n}\right)$. Let $b=b_{1} \vee \cdots \vee b_{n}$. Then $b \in T_{y}$ for all $y \in C$, so $C \subseteq V(b)$, hence $V(b)^{c} \subseteq U$. We also have that $b^{-}=b_{1}^{-} \wedge \cdots \wedge b_{n}^{-} \in T_{x}$. Let us prove that $V\left(b^{-}\right) \subseteq U$. If $z \in V\left(b^{-}\right)$, then $b^{-} \in T_{z}$, so $b \notin T_{z}$, since $T_{z}$ is proper. That is, $z \notin V(b)$, so $z \in U$. Thus, for any open subset $U$ of $X$ and any $x \in U$, we have got $b^{x} \in A$ such that $b^{x} \in T_{y}$ for all $y \notin U,\left(b^{x}\right)^{-} \in T_{x}$ and $V\left(\left(b^{x}\right)^{-}\right) \subseteq U$. It follows that $U=\bigcup_{x \in U} V\left(\left(b^{x}\right)^{-}\right)$, hence $U$ is open in the representation topology. Thus, we have proved that the topology on $X$ is generated by the family $V(a)_{a \in A}$. Let us now prove that the family $\left(T_{x}\right)_{x \in X}$ is strongly coprime. Let $x \in X$ and $a \in T_{x}$, i.e. $x \in V(a)$. Applying the above construction for $U=V(a)$, there is $b \in A$ such that $b^{-} \in T_{x}$ and $b \in T_{y}$ for all $y \notin V(a)$, so $b \in \bigcap\left\{T_{y} \mid y \in X\right.$ and $\left.a \notin T_{y}\right\}$. Hence, $0=b \odot b^{-} \in T_{x} \vee \bigcap\left\{T_{y} \mid y \in X\right.$ and $\left.a \notin T_{y}\right\}$, that is, $T_{x} \vee \bigcap\left\{T_{y} \mid y \in X\right.$ 
and $\left.a \notin T_{y}\right\}=A$. Apply now Proposition 4.4 and the fact that $X$ is compact to get (i).

(i) $\Leftrightarrow$ (iii) By Theorem 4.1 and Proposition 4.4, we have that the filters $\left(T_{x}\right)_{x \in X}$ canonically determine a completely regular representation of $A, \varphi: A \rightarrow \Gamma(X, F)$ iff the family $\left(T_{x}\right)_{x \in X}$ is strongly coprime and the topology on $X$ is generated by the family $V(a)_{a \in A}$. Now, applying The compactness theorem we obtain that the representation $\varphi$ is compact iff $\varphi(A)$ is compact in $(F, p, X)$ iff every maximal filter of $\varphi(A)$ is fixed. Applying now Lemma 3.6(i) and the fact that $A \cong \varphi(A)$, we get that every maximal filter of $\varphi(A)$ is fixed iff any maximal filter of $A$ contains a filter $T_{x}$ for some $x \in X$.

Applying Theorem 4.5, we prove the existence of a compact representation for any nontrivial BL-algebra $A$.

Proposition 4.6 The family $(O(M))_{M \in \operatorname{Max}(A)}$ canonically determines a compact representation of $A$.

Proof: We have that $\operatorname{Max}(A)$ is compact and Hausdorff and, applying Propositions 1.17(ii) and 1.18, it follows that the family $(O(M))_{M \in \operatorname{Max}(A)}$ is coprime. It remains to prove that $V(a)=\{M \in \operatorname{Max}(A) \mid a \in O(M)\}$ is open in $\operatorname{Max}(A)$ for all $a \in A$. Let $M \in V(a)$. Then $a \in O(M)$, so there is $b \notin M$ such that $a \vee b=1$. If $N \in d(b)$, then $b \notin N$ and $a \vee b=1$, so $a \in O(N)$, that is $N \in V(a)$. Hence, $M \in d(b) \subseteq V(a)$, so $V(a)$ is open.

Let $\left(F_{A}, p_{A}, \operatorname{Max}(A)\right)$ be the BL-sheaf space and $\varphi: A \rightarrow \Gamma\left(\operatorname{Max}(A), F_{A}\right)$ the canonical compact representation determined by the family $(O(M))_{M \in \operatorname{Max}(A)}$. Then $\left(F_{A}\right)_{M}=A / O(M)$ for all $M \in \operatorname{Max}(A), p_{A}: F_{A} \rightarrow \operatorname{Max}(A)$ is the canonical projection and $\varphi(a)=[a]$ for all $a \in A$, where $[a] \in \Gamma\left(\operatorname{Max}(A), F_{A}\right)$ is defined by $[a](M)=a / O(M)$ for all $M \in \operatorname{Max}(A)$.

Proposition $4.7 \varphi: A \cong \Gamma\left(\operatorname{Max}(A), F_{A}\right)$.

Proof: We have to prove that $\varphi(A)=\Gamma\left(\operatorname{Max}(A), F_{A}\right)$. Since $\varphi(A)$ is compact in $\left(F_{A}, p_{A}, \operatorname{Max}(A)\right)$, by Proposition 3.5 , it is sufficient to show that every global section is locally in $\varphi(A)$. Let $\sigma \in \Gamma\left(\operatorname{Max}(A), F_{A}\right)$. Then for all $M \in \operatorname{Max}(A), \sigma(M) \in A / O(M)$, so there is $a_{M} \in A$ such that $\sigma(M)=$ $a_{M} / O(M)=\left[a_{M}\right](M)=\varphi\left(a_{M}\right)(M)$, so $M \in\left[\sigma=\varphi\left(a_{M}\right)\right]$. Thus, there is a family $\left(a_{M}\right)_{M \in \operatorname{Max}(A)}$ of elements of $A$ and a family $\left(U_{M}=\left[\sigma=\varphi\left(a_{M}\right)\right]\right)_{M \in \operatorname{Max}(A)}$ of open sets of $\operatorname{Max}(A)$ such that $\left.\sigma\right|_{U_{M}}=\left.\varphi\left(a_{M}\right)\right|_{U_{M}}$ for all $M \in \operatorname{Max}(A)$. That is, $\sigma$ is locally in $\varphi(A)$.

\section{The equivalence between BL-algebras and com- pact local BL-sheaf spaces}

Let us denote by by $C L-B L-S h S p$ the full subcategory of $B L-S h S p$ whose objects are compact local BL-sheaf spaces. By Proposition 2.5, there is a sec- 
tion functor $\mathcal{S}: B L-S h S p \rightarrow B L$. Then, by composing $\mathcal{S}$ with the inclusion functor, we get a functor from $C L-B L-S h S p$ to $B L$, denoted by $\mathcal{S}$, too. In the sequel, we shall define a functor $\mathcal{T}: B L \rightarrow C L-B L-S h S p$ and we shall prove that the functors $\mathcal{S}, \mathcal{T}$ determine an equivalence between $C L-B L-S h S p$ and $B L$.

For any nontrivial BL-algebra $A$, let us define $\mathcal{T}(A)=\left(F_{A}, p_{A}, \operatorname{Max}(A)\right)$. By the previous section, $\left(F_{A}, p_{A}, \operatorname{Max}(A)\right)$ is a compact Bl-sheaf space. For any $M \in \operatorname{Max}(A)$, we have that the stalk at $M$ is $\left(F_{A}\right)_{M}=A / O(M)$. By Proposition 1.17(iii), $A / O(M)$ is a local BL-algebra, so $\left(F_{A}, p_{A}, \operatorname{Max}(A)\right)$ is a compact local BL-sheaf space.

Let $A$ and $B$ be nontrivial BL-algebras and $h: A \rightarrow B$ a BL-morphism. If $M$ is a maximal filter of $B$, then $h^{-1}(M)$ is a maximal filter of $A$, by Proposition 1.10. Let us define $\bar{h}: \operatorname{Max}(B) \rightarrow \operatorname{Max}(A)$ by $(\bar{h})(M)=h^{-1}(M)$ for any maximal filter $M$ of $B$.

Proposition 5.1 Let $\bar{h}: \operatorname{Max}(B) \rightarrow \operatorname{Max}(A)$ be the function defined above. Then

(i) $O(\bar{h}(M)) \subseteq h^{-1}(O(M)) \subseteq \bar{h}(M)$ for any maximal filter $M$ of $B$;

(ii) $\bar{h}$ is continuous.

Proof: (i) Let $a \in O(\bar{h}(M))$, so there is $b \notin \bar{h}(M)$ such that $a \vee b=1$. It follows that $h(a) \vee h(b)=1$ and $h(b) \notin M$, since $b \notin h^{-1}(M)$. That is, $h(a) \in O(M)$, hence $a \in h^{-1}(O(M))$. By Proposition 1.18(i), $O(M) \subseteq M$, hence $h^{-1}(O(M)) \subseteq h^{-1}(M)=\bar{h}(M)$.

(ii) Let $M \in \operatorname{Max}(B)$ and $V$ an open neighbourhood of $\bar{h}(M)$. We shall prove that there is an open neighbourhood $U$ of $M$ such that $\bar{h}(U) \subseteq V$, hence $\bar{h}$ is continuous at $M$. Since $\varphi: A \rightarrow\left(F_{A}, p_{A}, \operatorname{Max}(A)\right)$ is a completely regular representation of $A$, there is $a \in A$ such that $\varphi(a)(\bar{h}(M))=0 / O(\bar{h}(M))$ and $\varphi(a)(N)=1 / O(N)$ for all $N \in / V$. Hence, $a / O(\bar{h}(M))=0 / O(\bar{h}(M))$ and $a / O(N)=1 / O(N)$ for all $N \notin V$. Applying Proposition 1.7, we get that $a^{-} \in O(\bar{h}(M)$ and $a \in O(N)$ for all $N \notin V$. Since $O(\bar{h}(M)) \subseteq \bar{h}(M)$, we have that $a^{-} \in \bar{h}(M)$ and from the fact that $\bar{h}(M)$ is a maximal filter of $A$, it follows that $a \notin \bar{h}(M)$, hence $h(a) \notin M$. Thus, we have obtained that $M$ is an element of the basic open set $U=d(h(a))$ of $\operatorname{Max}(B)$. Let us prove that $\bar{h}(U) \subseteq V$. Suppose that there is $P \in U$ such that $\bar{h}(P) \notin V$. From $\bar{h}(P) \notin V$, it follows that $a \in O(\bar{h}(P))$, so, by $(\mathrm{i}), a \in \bar{h}(P)$, that is $h(a) \in P$. This contradicts the fact that $P \in U$. Thus, $\bar{h}(U) \subseteq V$.

Let $\left(\bar{h}^{-1}\left(F_{A}\right), q_{A}, \operatorname{Max}(B)\right)$ be the BL-sheaf space induced by $\bar{h}: \operatorname{Max}(B) \rightarrow$ $\operatorname{Max}(A)$ and $\left(F_{A}, p_{A}, \operatorname{Max}(A)\right)$ and $i: F_{A} \rightarrow \bar{h}^{-1}\left(F_{A}\right)$ the canonical morphism over $\bar{h}$. Since $\bar{h}: \operatorname{Max}(B) \rightarrow \operatorname{Max}(A)$ is continuous, we get that $(\bar{h}, i):\left(F_{A}, p_{A}, \operatorname{Max}(A)\right) \rightarrow\left(\bar{h}^{-1}\left(F_{A}\right), q_{A}, \operatorname{Max}(B)\right)$ is a morphism of BL-sheaf spaces. 
Proposition 5.2 For any maximal filter $M \in \operatorname{Max}(B)$, let us define $\psi_{M}$ : $\left(\bar{h}^{-1}\left(F_{A}\right)\right)_{M} \rightarrow\left(F_{B}\right)_{M}$, by $\psi_{M}(M, a / O(\bar{h}(M))=h(a) / O(M)$ for any $a \in A$. Then $\left(1_{\operatorname{Max}(B)}, \psi\right):\left(\bar{h}^{-1}\left(F_{A}\right), q_{A}, \operatorname{Max}(B)\right) \rightarrow\left(F_{B}, p_{B}, \operatorname{Max}(B)\right)$ is a morphism of BL-sheaf spaces.

Proof: Firstly, let us prove that $\psi_{M}$ is well-defined. Let $a, b \in A$ such that $a / O(\bar{h}(M))=b / O(\bar{h}(M))$. It follows that $(a \rightarrow b) \odot(b \rightarrow a) \in O(\bar{h}(M))$, that is $(a \rightarrow b) \odot(b \rightarrow a) \in h^{-1}(O(M))$, so $(h(a) \rightarrow h(b)) \odot(h(b) \rightarrow h(a)) \in O(M)$. Thus, $h(a) / O(M)=h(b) / O(M)$. Now, we shall apply Proposition 2.4 to get that $\left(1_{\operatorname{Max}(B)}, \psi\right)$ is a morphism of BL-sheaf spaces. By the definition of $\psi$, it follows immediately that $p_{B} \circ \psi=q_{A}$ and that $\psi_{M}:\{M\} \times A / O(\bar{h}(M)) \rightarrow$ $B / O(M)$ is a BL-morphism for all $M \in \operatorname{Max}(B)$. It remains to prove that $\psi$ is continuous. By Proposition 2.1(vi), it is sufficient to prove that $\psi$ is open. Since the family $\{d(a) \mid a \in A\}$ is a basis for $\operatorname{Max}(A)$, by Proposition 2.1(iv), Proposition 4.7, it follows that a basis for the topology of $F_{A}$ is the family $\{[c](d(a)) \mid a, c \in A\}$. We get that a basis for $\bar{h}^{-1}\left(F_{A}\right)$ is the family $\{(d(b) \times$ $\left.[c](d(a))) \cap \bar{h}^{-1}(A) \mid a, c \in A, b \in B\right\}$. A basic open set in $\bar{h}^{-1}(A)$ is $(d(b) \times$ $[c](d(a))) \cap \bar{h}^{-1}(A)=\{(M, c / O(\bar{h}(M))) \mid M \in \operatorname{Max}(B), b \notin M, a \in \bar{h}(M)\}=$ $\{(M, c / O(\bar{h}(M))) \mid M \in \operatorname{Max}(B), b \in M, h(a) \notin M\}$, where $a, c \in A, b \in B$. It follows that $\psi\left((d(b) \times[c](d(a))) \cap \bar{h}^{-1}(A)\right)=\{\psi(M, c / O(\bar{h}(M))) \mid M \in$ $\operatorname{Max}(B), b \notin M, h(a) \notin M\}=\{h(c) / O(M) \mid M \in \operatorname{Max}(B), b \notin M, h(a) \notin M\}=$ $[h(c)](d(b) \cap d(h(a)))$, which is open in $F_{B}$. Hence, $\psi$ is open.

Hence, for a BL-morphism $h: A \rightarrow B$, we have got the morphisms of BL-sheaf spaces $(\bar{h}, i):\left(F_{A}, p_{A}, \operatorname{Max}(A)\right) \rightarrow\left(\bar{h}^{-1}\left(F_{A}\right), q_{A}, \operatorname{Max}(B)\right)$ and $\left(1_{\operatorname{Max}(B)}, \psi\right)$ : $\left(\bar{h}^{-1}\left(F_{A}\right), q_{A}, \operatorname{Max}(B)\right) \rightarrow\left(F_{B}, p_{B}, \operatorname{Max}(B)\right)$. We define $\mathcal{T}(h)=\left(1_{\operatorname{Max}(B)}, \psi\right) \circ$ $(\bar{h}, i)=\left(\bar{h}, \alpha_{h}\right): \mathcal{T}(A) \rightarrow \mathcal{T}(B)$, where $\alpha_{h}=\psi \circ i$.

Thus, we finished the definition of the functor $\mathcal{T}: B L \rightarrow C L-B L-S h S p$.

Proposition $5.3 \mathcal{S} \circ \mathcal{T} \cong 1_{B L}$.

Proof: For any BL-algebra $A$, we have that $(\mathcal{S} \circ \mathcal{T})(A)=\Gamma\left(\operatorname{Max}(A), F_{A}\right)$ and for any BL-morphism $h: A \rightarrow B,(\mathcal{S} \circ \mathcal{T})(h)=\mathcal{S}\left(\bar{h}, \alpha_{h}\right)=\alpha_{h \#}$, where $\alpha_{h \#}: \Gamma\left(\operatorname{Max}(A), F_{A}\right) \rightarrow \Gamma\left(\operatorname{Max}(B), F_{B}\right)$ is the BL-morphism induced by $\alpha_{h}$. By Proposition 4.7, we have an isomorphism $\varphi_{A}: A \cong \Gamma\left(\operatorname{Max}(A), F_{A}\right)$ for any non-trivial BL-algebra $A$. Let us prove that $\varphi=\left(\varphi_{A}\right)_{A \in O b(B L)}: 1_{B L} \cong \mathcal{S} \circ \mathcal{T}$ is a natural transformation. For any $a \in A$ and $M \in \operatorname{Max}(B)$, we have that $\left(\left(\alpha_{h \#} \circ\right.\right.$ $\left.\left.\varphi_{A}\right)(a)\right)(M)=\left(\left(\alpha_{h \#}\right)[a]\right)(M)=\left(\alpha_{h M}\right)([a](\bar{h}(M)))=\left(\alpha_{h M}\right)(a / O(\bar{h}(M)))=$ $h(a) / O(M)$ and $\left(\left(\varphi_{B} \circ h\right)(a)\right)(M)=[h(a)](M)=h(a) / O(M)$. Thus, $(\mathcal{S} \circ$ $\mathcal{T})(h) \circ \varphi_{A}=\varphi_{B} \circ h$. Hence, $\varphi: 1_{B L} \cong \mathcal{S} \circ \mathcal{T}$ is a natural isomorphism.

Proposition 5.4 $\mathcal{T} \circ \mathcal{S} \cong 1_{C L-B L-S h S p}$. 
Proof: Let $(F, p, X)$ be a compact local BL-sheaf space and $A=\Gamma(X, F)$. Then $(\mathcal{T} \circ \mathcal{S})(F, p, X)=\mathcal{T}(A)=\left(F_{A}, p_{A}, \operatorname{Max}(A)\right)$. Let $\mathbf{n}: X \rightarrow \operatorname{Max}(A)$ the function that associates with any $x \in X$ the unique maximal filter $M$ of $A$ such that $K_{x} \subseteq M$. By Proposition 3.17, $\mathbf{n}$ is a homeomorphism. Let $x \in X$ and $M=\mathbf{n}(x)$, so $\mathbf{m}(M)=x$. Then, by Proposition 3.12, we get that $O(M)=K_{\mathbf{m}(M)}$, that is $O(\mathbf{n}(x))=K_{x}$. Applying now Proposition 3.2(iv), it follows that $A / O(\mathbf{n}(x)) \cong F_{x}$. If $\alpha_{x}: A / O(\mathbf{n}(x)) \rightarrow F_{x}$ is this isomorphism, then $\alpha_{x}(\sigma / O(\mathbf{n}(x)))=\sigma(x)$ for all $\sigma \in A$. Let us prove that $\alpha: F_{A} \rightarrow F$ is a morphism over $\mathbf{n}$. Let $D$ be an open subset of $\operatorname{Max}(A)$ and $t \in \Gamma\left(D, F_{A}\right)$. We have to prove that the function $\alpha_{\#}^{D}(t): \mathbf{n}^{-1}(D) \rightarrow F$, defined by $\alpha_{\#}^{D}(t)(x)=$ $\alpha_{x}(t(\mathbf{n}(x)))$ for any $x \in \mathbf{n}^{-1}(D)$, is continuous. Since $\left(F_{A}, p_{A}, \operatorname{Max}(A)\right)$ is compact and $t$ is a section over an open subset $D$ of $\operatorname{Max}(A)$, we can apply Proposition 3.2(ii) to get an open covering $\left(D_{i}\right)_{i \in I}$ of $D$ and a family $\left(t_{i}\right)_{i \in I}$ of sections from $\Gamma\left(\operatorname{Max}(A), F_{A}\right)$ such that $\left.t\right|_{D_{i}}=\left.t_{i}\right|_{D_{i}}$ for all $i \in I$. Applying now Proposition 4.7, we obtain a family $\left(\sigma_{i}\right)_{i \in I}$ of sections from $\Gamma(X, F)$ such that $t_{i}=\left[\sigma_{i}\right]$ for all $i \in I$. Let $x \in \mathbf{n}^{-1}(D)$, that is $\mathbf{n}(x) \in D$. Then, there is $k \in I$ such that $\mathbf{n}(x) \in D_{k}$, so $t(\mathbf{n}(x))=\left[\sigma_{k}\right](\mathbf{n}(x))=\sigma_{k} / O(\mathbf{n}(x))$. It follows that $\alpha_{\#}^{D}(t)(x)=\alpha_{x}\left(\sigma_{k} / O(\mathbf{n}(x))\right)=\sigma_{k}(x)$. Let $V \subseteq F$ be an open neighbourhood of $\sigma_{k}(x)$ and $U=\mathbf{n}^{-1}\left(D_{k}\right) \cap \sigma_{k}^{-1}(V)$. Then $U \subseteq \mathbf{n}^{-1}(D)$ is an open neighbourhood of $x$. If $y \in U$, then $\mathbf{n}(y) \in D_{k}$ and $\sigma_{k}(y) \in V$. It follows that $t(\mathbf{n}(y))=\sigma_{k} / O(\mathbf{n}(y))$, hence $\alpha_{\#}^{D}(t)(y)=\sigma_{k}(y) \in V$. Thus, $\alpha_{\#}^{D}(t)(U) \subseteq V$. Hence, we have proved that for any $x \in \mathbf{n}^{-1}(U)$ and for any open neighbourhood $V$ of $\alpha_{\#}^{D}(t)(x)$ there is an open neighbourhood $U$ of $x$ such that $\alpha_{\#}^{D}(t)(U) \subseteq V$. That is, $\alpha_{\#}^{D}(t)$ is continuous.

Hence, $(\mathbf{n}, \alpha):\left(F_{A}, p_{A}, \operatorname{Max}(A)\right) \rightarrow(F, p, X)$ is an isomorphism of BL-sheaf spaces. Let $\lambda: \mathcal{T} \circ \mathcal{S} \rightarrow 1_{C L-B L-S h S p}$, where $\left.\lambda_{(F, p, X)}\right)=(\mathbf{n}, \alpha)$. It remains to prove that $\lambda$ is a natural transformation. Let $(f, \beta):(F, p, X) \rightarrow(G, q, Y)$ be a morphism of BL-sheaf spaces, $A=\Gamma(X, F)$ and $B=\Gamma(Y, G)$. Let $\left.\left.\lambda_{(F, p, X)}\right)=\left(\mathbf{n}_{\mathbf{1}}, \alpha_{1}\right), \lambda_{(G, q, Y)}\right)=\left(\mathbf{n}_{\mathbf{2}}, \alpha_{2}\right)$. We have that $(\mathcal{T} \circ \mathcal{S})(f, \beta)=\left(\overline{\beta_{\#}}, \theta\right)$, where $\beta_{\#}: A \rightarrow B, \beta_{\#}(\sigma)(y)=\beta_{y}(\sigma(f(y)))$ for all $\sigma \in A$ and $y \in Y$, $\overline{\beta_{\#}}: \operatorname{Max}(B) \rightarrow \operatorname{Max}(A), \overline{\beta_{\#}}(M)=\left(\beta_{\#}\right)^{-1}(M)$ and $\theta=\left(\theta_{M}\right)_{M \in \operatorname{Max}(B)}$, $\theta_{M}: A / O\left(\overline{\beta_{\#}}(M)\right) \rightarrow B / O(M), \theta_{M}\left(\sigma /\left(\overline{\beta_{\#}}(M)\right)\right)=\beta_{\#}(\sigma) / O(M)$ for all $\sigma \in A$. We have to prove that $(f, \beta) \circ\left(\mathbf{n}_{\mathbf{1}}, \alpha_{1}\right)=\left(\mathbf{n}_{\mathbf{2}}, \alpha_{2}\right) \circ\left(\overline{\beta_{\#}}, \theta\right)$, that is $\left(\mathbf{n}_{\mathbf{1}} \circ f, \beta \circ \alpha_{1}\right)=$ $\left(\overline{\beta_{\#}} \circ \mathbf{n}_{\mathbf{2}}, \alpha_{2} \circ \theta\right)$. Let $y \in Y$. If $\sigma \in K_{f(y)}$, then $\sigma(f(y))=1_{f(y)}$, hence $\beta_{\#}(\sigma)(y)=\beta_{y}\left(\sigma(f(y))=\beta_{y}\left(1_{f(y)}\right)=1_{y}\right.$, so $\beta_{\#}(\sigma) \in K_{y} \subseteq \mathbf{n}_{\mathbf{2}}(y)$, that is $\sigma \in\left(\beta_{\#}\right)^{-1}\left(\mathbf{n}_{\mathbf{2}}(y)\right)=\overline{\beta_{\#}}\left(\mathbf{n}_{\mathbf{2}}(y)\right)$. Thus, we have proved that $\overline{\beta_{\#}}\left(\mathbf{n}_{\mathbf{2}}(y)\right.$ is a maximal filter of $A$ that contains $K_{f(y)}$. But $\mathbf{n}_{\mathbf{1}}(f(y))$ is the unique maximal filter of $A$ that contains $K_{f(y)}$. Hence, we must have $\left(\beta_{\#}\right)^{-1}\left(\mathbf{n}_{\mathbf{2}}(y)\right)=\mathbf{n}_{\mathbf{1}}(f(y))$. Let us prove now that $\beta \circ \alpha_{1}=\alpha_{2} \circ \theta$. Let $y \in Y$ and $\sigma \in A$. Then $(\beta \circ$ $\left.\alpha_{1}\right)_{y}\left(\sigma / O\left(\mathbf{n}_{\mathbf{1}}(f(y))\right)=\beta_{y}(\sigma(f(y)))=\beta_{\#}(\sigma)(y)\right.$ and $\left(\alpha_{2} \circ \theta\right)_{y}\left(\sigma / O\left(\overline{\beta_{\#}}\left(\mathbf{n}_{\mathbf{2}}(y)\right)\right)=\right.$ $\alpha_{2 y}\left(\beta_{\#}(\sigma) / O\left(\mathbf{n}_{2}(y)\right)\right)=\beta_{\#}(\sigma)(y)$.

Thus, we have got 
Theorem 5.5 The functors $\mathcal{S}: C L-B L-S h S p \rightarrow B L$ and $\mathcal{T}: B L \rightarrow$ $C L-B L-S h S p$ establish an equivalence between the category of nontrivial BL-algebras and the category of compact local BL-sheaf spaces.

As a consequence, we get the corresponding result for MV-algebras.

Corollary 5.6 [7] The functor from the category of compact local MV-sheaf spaces to the category of nontrivial MV-algebras, obtained by assigning to each compact local MV-sheaf space the MV-algebra of global sections, determines an equivalence between these categories.

\section{Acknowledgement}

The research of Laurenţiu Leuştean has been partially supported by a Marie Curie Fellowship of the European Community Programme Improving the $\mathrm{Hu}-$ man Research Potential and the Socio-economic Knowledge Base under contract number HPTM-CT-2000-00093 at BRICS, Basic Research in Computer Science (www.brics.dk), funded by the Danish National Research Foundation.

\section{References}

[1] R. Cignoli, I.M.L. D'Ottaviano, D. Mundici, Algebraic Foundations of manyvalued Reasoning, Kluwer Academic Publishers, Dordrecht, 2000.

[2] W. Cornish, Normal lattices, Journal of the Australian Mathematical Society, 14(1972), 200-215.

[3] B. A. Davey, Sheaf spaces and sheaves of universal algebras, Mathematische Zeitschrift, 134(1973), 275-290.

[4] A. Di Nola, G. Georgescu, A. Iorgulescu, Pseudo-BL algebras: Part I, International Journal of Multiple-Valued Logic, to appear.

[5] A. Di Nola, G. Georgescu, A. Iorgulescu, Pseudo-BL algebras: Part II, International Journal of Multiple-Valued Logic, to appear.

[6] A. Di Nola, G. Georgescu, L. Leuştean, Boolean products of BL-algebras, Journal of Mathematical Analysis and Applications, 251(2000), 106-131.

[7] A. Filipoiu, G. Georgescu, Compact and Pierce representations of MValgebras, Revue Roumaine de Mathématiques Pures et Appliquées, Tome 40, 7-8(1995), 599-618.

[8] I. Gelfand, A. Kolmogoroff, On rings of continuous functions on topological spaces, Dokl. Akad. Nauk. SSSR, 22(1939), 11-15.

[9] G. Georgescu, L. Leuştean, Some classes of pseudo-BL algebras, Journal of the Australian Mathematical Society, to appear. 
[10] G. Georgescu, I. Voiculescu, Some abstract maximal-ideal like spaces, Algebra Universalis, 26(1989), 90-102.

[11] P. Hájek, Metamathematics of Fuzzy Logic, Kluwer Academic Publishers, Dordrecht, 1998.

[12] L. Leuştean, The prime and maximal spectra and the reticulation of BLalgebras, submitted.

[13] C. J. Mulvey, Compact ringed spaces, Journal of Algebra, 52(1978), 411436.

[14] C. J. Mulvey, Representations of rings and modules, in "Applications of sheaves", Lecture Notes in Mathematics 753, Springer Verlag, 1979.

[15] C. J. Mulvey, A generalisation of Gelfand duality, Journal of Algebra, 56(1979), 499-505.

[16] Y.S. Pawar, Characterizations of normal lattices, Indian Journal of Pure and Applied Mathematics, 24(1993), 651-656.

[17] H. Schubert, Topology, Macdonald \& Co.(Publishers) Ltd., 1968.

[18] H. Simmons, Reticulated rings, Journal of Algebra, 66(1980), 169-192.

[19] R. S. Swan, The Theory of Sheaves, Chicago Lectures in Mathematics, The University of Chicago Press, 1964.

[20] B. R. Tennison, Sheaf Theory, London Mathematical Society Lecture Notes Series 20, Cambridge University Press, 1975.

[21] E. Turunen, BL-algebras of Basic Fuzzy Logic, Mathware and Soft Computing, 6(1999), 49-61.

[22] Esko Turunen, Salvatore Sessa, Local BL-algebras, International Journal of Multiple-Valued Logic, 6(2001), 229-249.

[23] H. Wallman, Lattices and topological spaces, Annals of Mathematics, 39(1938), 112-126. 


\section{Recent BRICS Report Series Publications}

RS-02-23 Antonio Di Nola and Laurenţiu Leuştean. Compact Representations of BL-Algebras. May 2002. 25 pp.

RS-02-22 Mogens Nielsen, Catuscia Palamidessi, and Frank D. Valencia. On the Expressive Power of Concurrent Constraint Programming Languages. May 2002. 34 pp.

RS-02-21 Zoltán Ésik and Werner Kuich. Formal Tree Series. April 2002. $66 \mathrm{pp}$.

RS-02-20 Zoltán Ésik and Kim G. Larsen. Regular Languages Definable by Lindström Quantifiers (Preliminary Version). April 2002. $56 \mathrm{pp}$.

RS-02-19 Stephen L. Bloom and Zoltán Ésik. An Extension Theorem with an Application to Formal Tree Series. April 2002. 51 pp.

RS-02-18 Gerth Stølting Brodal and Rolf Fagerberg. Cache Oblivious Distribution Sweeping. April 2002. To appear in 29th International Colloquium on Automata, Languages, and Programming, ICALP '02 Proceedings, LNCS, 2002.

RS-02-17 Bolette Ammitzbøll Madsen, Jesper Makholm Nielsen, and Bjarke Skjernaa. On the Number of Maximal Bipartite Subgraphs of a Graph. April 2002. 7 pp.

RS-02-16 Jiří Srba. Strong Bisimilarity of Simple Process Algebras: Complexity Lower Bounds. April 2002. To appear in 29th International Colloquium on Automata, Languages, and Programming, ICALP '02 Proceedings, LNCS, 2002.

RS-02-15 Jesper Makholm Nielsen. On the Number of Maximal Independent Sets in a Graph. April 2002. 10 pp.

RS-02-14 Ulrich Berger and Paulo B. Oliva. Modified Bar Recursion. April 2002. 23 pp.

RS-02-13 Gerth Stølting Brodal, Rune B. Lyngsø, Anna Östlin, and Christian N. S. Pedersen. Solving the String Statistics Problem in Time $O(n \log n)$. March 2002. To appear in 29th International Colloquium on Automata, Languages, and Programming, ICALP '02 Proceedings, LNCS, 2002. 